\title{
German National Guideline for Treating Chronic Respiratory Failure with Invasive and Non-Invasive Ventilation - Revised Edition 2017: Part 2
}

\author{
Wolfram Windisch ${ }^{\mathrm{a}, \mathrm{b}}$ Jens Geiseler ${ }^{\mathrm{c}}$ Karsten Simon ${ }^{\mathrm{d}}$ Stephan Walterspacher ${ }^{\mathrm{b}, \mathrm{e}}$ \\ Michael Dreher $^{f}$ on behalf of the Guideline Commission \\ ${ }^{a}$ Department of Pneumology, Cologne Merheim Hospital, Kliniken der Stadt Köln gGmbH, Cologne, Germany; \\ ${ }^{b}$ Faculty of Health/School of Medicine, Witten/Herdecke University, Witten, Germany; ${ }^{\mathrm{C}}$ Medical Clinic IV, Pneumology, \\ Sleep medicine and Mechanical Ventilation, Paracelsus-Klinik Marl, Marl, Germany; ${ }^{d}$ Fachkrankenhaus Kloster

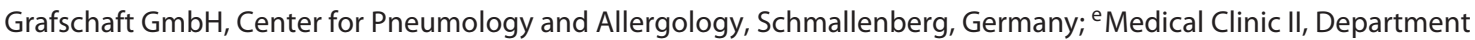 \\ of Pneumology, Cardiology and Intensive Care Medicine, Klinikum Konstanz, Konstanz, Germany; ${ }^{\mathrm{f} D i v i s i o n}$ of \\ Pneumology, University Hospital RWTH Aachen, Aachen, Germany
}

\section{Keywords}

Home mechanical ventilation - Non-invasive ventilation . Invasive ventilation - Chronic respiratory failure - Weaning • End-of-life

\begin{abstract}
Today, invasive and non-invasive home mechanical ventilation have become a well-established treatment option. Consequently, in 2010, the German Respiratory Society (DGP) has leadingly published the guidelines on "Non-Invasive and Invasive Mechanical Ventilation for Treatment of Chronic Respiratory Failure." However, continuing technical evolutions, new scientific insights, and health care developments require an extensive revision of the guidelines. For this reason, the updated guidelines are now published. Thereby, the existing chapters, namely technical issues, organizational structures in Germany, qualification criteria, disease-specific recommendations including special features in pediatrics as well as ethical aspects and palliative care, have been updated according to the current literature and the health care developments in Germany. New chapters added to the guidelines include the topics of home mechanical ventila-
\end{abstract}

tion in paraplegic patients and in those with failure of prolonged weaning. In the current guidelines, different societies as well as professional and expert associations have been involved when compared to the 2010 guidelines. Importantly, disease-specific aspects are now covered by the German Interdisciplinary Society of Home Mechanical Ventilation (DIGAB). In addition, societies and associations directly involved in the care of patients receiving home mechanical ventilation have been included in the current process. Importantly, associations responsible for decisions on costs in the health care system and patient organizations have now been involved.

(c) 2018 S. Karger AG, Basel

S. Walterspacher and M. Dreher contributed equally to this work. Guideline Commission: W. Windisch, M. Dreher, J. Geiseler, K. Siemon, J. Brambring, D. Dellweg, B. Grolle, S. Hirschfeld, T. Köhnlein, U. Mellies, S. Rosseau, B. Schönhofer, B. Schucher, A. Schütz, H. Sitter, S. Stieglitz, J. Storre, M. Winterholler, P. Young, S. Walterspacher.

This is part 2 of the German National Guideline for Treating Chronic Respiratory Failure with Invasive and Non-Invasive Ventilation - Revised Edition (Chapters 9-16). For part 1 (Chapters 1-8), see Respiration 2018, DOI: 10.1159/000488667.

\section{KARGER}

(C) 2018 S. Karger AG, Basel

E-Mail karger@karger.com

www.karger.com/res
Prof. Dr. Wolfram Windisch

Department of Pneumology, Cologne Merheim Hospital

Kliniken der Stadt Köln gGmbH, Faculty of Health/School of Medicine

Witten/Herdecke University, Ostmerheimer Strasse 200, DE-51109 Köln (Germany)

E-Mail windischw@kliniken-koeln.de 


\section{Obstructive Airway Diseases}

\subsection{What Is the Significance of Non-Invasive}

Ventilation in Chronic Obstructive Pulmonary

Disease?

In comparison to the prescription of NIV for acute respiratory failure, there are only a few studies with sufficient quality criteria to support the long-term use of HMV via NIV for COPD patients with chronic respiratory failure [231,232]. NIV is the primary therapy option for chronic respiratory failure arising from COPD. Nowadays, long-term invasive ventilation via a tracheostoma is only applied under exceptional circumstances, predominantly after unsuccessful prolonged weaning (Group 3c, see Ch. 8.1) [152].

\subsubsection{What Are the Indications for Long-Term}

Non-Invasive Ventilation in Chronic Obstructive

Pulmonary Disease Patients?

The most important criterion for long-term NIV is either symptomatic chronic respiratory insufficiency or persistent hypercapnia following an acute NIV-dependent exacerbation requiring hospitalisation. A period of nicotine abstinence before NIV initiation is strongly recommended [233].

Indication criteria (at least 1 criterion must be fulfilled) (Fig. 3):

- Chronic diurnal hypercapnia with a $\mathrm{PaCO}_{2} \geq 50 \mathrm{~mm}$ $\mathrm{Hg}$.

- Nocturnal hypercapnia with a $\mathrm{PaCO}_{2} \geq 55 \mathrm{~mm} \mathrm{Hg}$.

- Mild diurnal hypercapnia with 46-50 $\mathrm{mm} \mathrm{Hg}$ and an increase in $\mathrm{PtcCO}_{2} \geq 10 \mathrm{~mm} \mathrm{Hg}$ during sleep.

- When persistent hypercapnia $\left(\mathrm{PaCO}_{2}>53 \mathrm{~mm} \mathrm{Hg}\right)$ is present at least 14 days after finishing acute ventilation therapy for acute respiratory acidosis [234].

- When decannulation after prolonged weaning is only possible with the help of NIV, and this is necessary for long-term monitoring of symptoms and prevention of hypercapnia, even after discharge from hospital (Weaning Category 3b).

The evaluation of "typical symptoms" always needs clinical appraisal, whereby additional criteria such as the progress of lung function, physical performance, and the patient's wishes are taken into consideration.

Patients with mild daytime hypercapnia $\left(\mathrm{PaCO}_{2} 45-\right.$ $50 \mathrm{~mm} \mathrm{Hg}$ ) require nocturnal diagnostic tests. Repetitive nocturnal measurements of blood gases are disruptive to sleep and only represent snapshots of the blood gas profile [25]. If transcutaneous $\mathrm{PCO}_{2}$ measurements are not available, a morning blood gas analysis directly after com- pletion of the nightly sleep can be useful for evaluating the indication for mechanical ventilation. Moreover, elevated bicarbonate values can be suggestive of nocturnal hypercapnia and hence serve as an indication for further clarification. Polygraphy or polysomnography are options for documenting the course of $\mathrm{PCO}_{2}$ in a sleep stage-dependent manner. An indication for NIV exists when mild diurnal hypercapnia is accompanied by a rise in $\mathrm{PtcCO}_{2}$ to $\geq 10 \mathrm{~mm} \mathrm{Hg}$ during nocturnal sleep. Repeated severe exacerbations with respiratory acidosis $(\mathrm{pH}$ $<7.35$ ) should be closely monitored for the continued existence of chronic hypercapnia. No scientific evidence exists to support the initiation of NIV immediately after a period of acute hypercapnia [235]. In contrast to this, the prognosis improves after ventilation-dependent respiratory acidosis if hypercapnia with a $\mathrm{PaCO}_{2}>53 \mathrm{~mm} \mathrm{Hg}$ persists for more than 14 days after completing the acute ventilation phase, and long-term NIV is then implemented [234]. Therefore, following an acute exacerbation with respiratory acidosis, patients who are discharged from hospital without home NIV should be re-evaluated by a private respiratory physician or the ventilation centre with respect to persistent diurnal hypercapnia $\left(\mathrm{PaCO}_{2}\right.$ $>53 \mathrm{~mm} \mathrm{Hg}$ ). More importantly, a specialised pneumological follow-up can essentially help to avoid rehospitalisation [236].

Recent scientific studies show that patients with an acute deterioration in chronic hypercapnia (acute on chronic) profit considerably from the initiation of longterm NIV. In contrast, patients who become hypercapnic and ventilation-dependent during the exacerbation phase, but afterwards become normocapnic again without further ventilatory support, do not benefit from the application of long-term NIV.

\subsubsection{How Is Non-Invasive Ventilation Carried}

Out on a Practical Level in Chronic Obstructive

Pulmonary Disease Patients?

The aim of the ventilation therapy is to normalise $\mathrm{PaCO}_{2}$ during ventilation and during ventilation-free intervals for optimal treatment success $[237,238]$. The best therapeutic effects of ventilation were achieved under experimental conditions through the use of assist-controlled ventilation modes with inspiratory pressures ranging from 20 to $40 \mathrm{mbar}[23,60,74-77,239,240]$. This so-called high-intensity NIV technique is defined as NIV with the physiological goal of maximally reducing $\mathrm{PCO}_{2}$ according to subjective tolerance. A detailed description of the high-intensity NIV technique can be found in the following references $[23,60,74-77,239-241]$. Empirical 
Fig. 3. Algorithm for treatment of chronic hypercapnic respiratory insufficiency in COPD. Stepwise approach to NIV therapy in COPD patients.

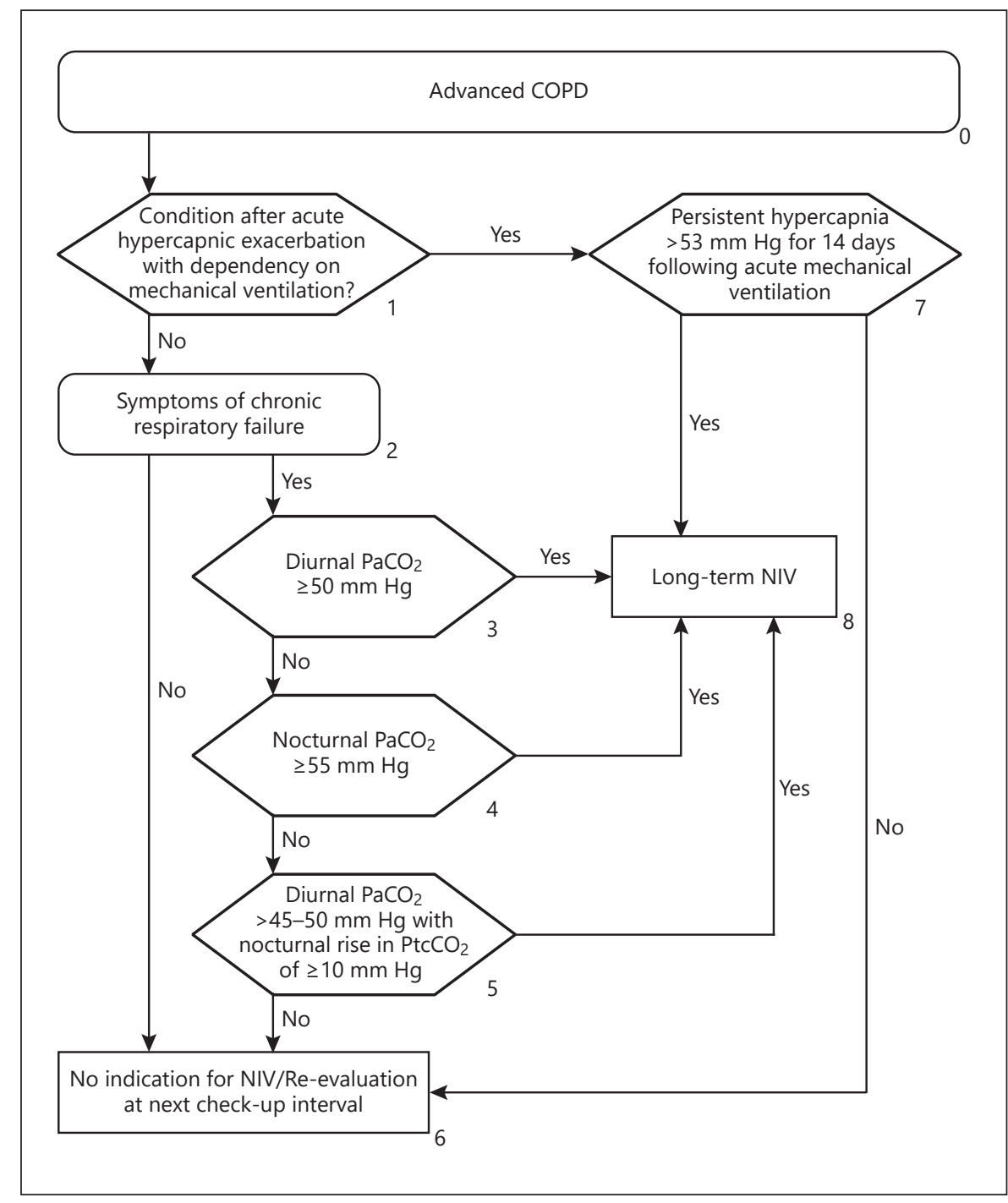

evidence suggests that ventilation presets are not applicable to all patients; the ventilation should therefore take place with a ventilation pressure that lies as closely as possible to the therapeutically required pressure but is still tolerated by the patient. However, it is necessary to allow the patient sufficient time to adapt to NIV, since successful initiation of high-intensity NIV can take longer than NIV in assisted mode without the goal of maximally reducing $\mathrm{PaCO}_{2}[23,60,74-77,239]$.

A rapid rise in inspiratory pressure (within 0.1-0.2 s) is best tolerated by patients. The use of PEEP/EPAP in assisted or assisted-controlled ventilation prevents upper airway collapse during sleep, which is especially useful in comorbid obstructive sleep apnoea syndrome (OSAS).

Treating Chronic Respiratory Failure with Invasive and Non-Invasive Ventilation
9.1.3 What Are the Expected Effects of Ventilation on Patients with Stable Chronic Hypercapnia?

Home NIV appears to be successful with respect to physiological parameters such as spontaneous breathing frequency, volume trauma, blood gases, lung function $[23,39,60,75-77,242-245]$, as well as cachexia [246] and breathing patterns [23]. Following initiation of NIV, COPD patients show an alleviation of their dyspnoea [23, $42,247]$. Other studies have demonstrated that home NIV improves physical performance, as measured by the 6-minute walking test [248]. This effect can be strengthened when NIV is combined with pulmonary rehabilitation [249-252]. Furthermore, the use of NIV during physical activity is associated with improvements in oxygenation, resilience, and dyspnoea [253, 254]. One par- 
ticular controlled study was able to show that the longterm use of NIV significantly improved sleep quality in COPD patients [243], although this effect could not be verified in a meta-analysis of 3 clinical studies [248]. Studies using the SRI questionnaire demonstrate a clear gain in health-related quality of life $[23,42,44,77,78$, 245], while the use of NIV is associated with a reduction in hospital admissions and inpatient treatment costs [242, 255-258].

In earlier controlled prospective studies, no clinically relevant advantages could be found for the use of NIV in chronically stable COPD patients [259, 260]. However, these studies had considerable flaws in terms of design, applied technique, patient selection, sample size, or observation period, meaning no real information about the actual therapeutic benefits of NIV could be gained from the findings [261-263]. Two smaller studies with an observation period of over 12 months could not show any survival benefits for mechanically ventilated patients $[242,264]$. This finding is in contrast to that of a retrospective data analysis [245] as well as that of a prospective study which demonstrated a statistically significant reduction in the mortality of patients who had undergone 2 years of long-term NIV with an average of $4.5 \mathrm{~h}$ per day [265]. The most comprehensive prospective randomisedcontrolled study with mortality as the primary target parameter was able to demonstrate in a cohort of 195 stable, chronically hypercapnic COPD patients that the 1-year mortality rate could be significantly reduced through long-term home NIV, that is, when a marked reduction in hypercapnia was achieved [78]. This requires the application of an inspiratory ventilation pressure beyond 20 mbar as well as the daily use of NIV over a period of at least $6 \mathrm{~h}$. Moreover, a significant improvement in physical performance and health-related quality of life could be established within this setting. The decision to use NIV on a long-term therapy basis lies with the treating physician and should obviously take patient-specific factors into account [266].

\subsubsection{What Are the Effects of Long-Term}

Non-Invasive Ventilation on Cardiac Function?

One particular physiological study found a reduction in cardiac output during parallel use of NIV, especially when high ventilation pressures were employed (highintensity NIV) [240]. It is therefore imperative that caution is exercised in patients with pre-existing cardiac insufficiency. It is, however, assumed that a reduction in cardiac volume also reflects in a favourable sense a decrease in the work of breathing [267]. Another study showed that long-term NIV in COPD patients led to a reduction in elevated proBNP values over time [268]. Finally, a recent randomised cross-over study reported that long-term NIV does not have a detrimental effect on cardiac function if high-intensity NIV is implemented rather than low-intensity NIV with low ventilation pressures and an assisted ventilation mode [269].

\subsubsection{What Particularly Needs to Be Observed for}

Long-Term Non-Invasive Ventilation of Chronic

Obstructive Pulmonary Disease Patients?

The initiation of NIV in patients with advanced COPD requires a high amount of motivation and cooperation from the patient's end and is a particular challenge for the therapy team. It can therefore take well up to 2 weeks on the hospital ward before stable treatment conditions are achieved $[42,75,270]$. This time frame is justified for the often elderly, multimorbid patients in order to optimally adapt both the ventilation mode and mask to the needs of the patient and to guarantee long-term therapy adherence [248].

\section{Recommendations}

- Non-invasive ventilation is the primary therapeutic option for home mechanical ventilation of chronic obstructive pulmonary disease patients with chronic respiratory failure.

- Home non-invasive ventilation must be initiated when the patient presents with chronic hypercapnia in combination with the typical symptoms of respiratory failure.

- Home non-invasive ventilation must be initiated when hypercapnia persists for more than 2 weeks after completing acute ventilation therapy for acute respiratory acidosis.

- The aim of the ventilation therapy is to normalise $\mathrm{PaCO}_{2}$; this may require sufficiently high ventilation intensity with effective ventilation pressures as well as the use of controlled ventilation modes.

\subsection{Cystic Fibrosis}

In addition to the above-described aspects of direct therapy for respiratory pump insufficiency in COPD patients, the improvement in gas exchange, enhancement of physical capabilities, and improvement in quality of life can, in combination with physiotherapy, facilitate the coughing-up of thick mucous secretions [271]. A recent study demonstrated an association between short NIV intervals and improved physical performance, more economical breathing, better lung function, and improved tissue oxygenation [272]. These effects were presumably achieved through the temporary reduction in respiratory muscle loading; direct effects on cystic fibrosis-related bronchiolitis appears less likely. At least 1 year of NIV appears to stabilise lung function in children in far-ad- 
vanced stages of the disease [273]. A tendency towards improved gas exchange during sleep has also been shown [274]. The positive effects of long-term NIV on exacerbation frequency, disease course, sleep quality, and healthrelated quality of life have been postulated a number of times, but should also be verified in larger studies with sufficient quality criteria [275].

\subsection{Bronchiectasis}

Currently, there are only a few relevant studies but no valid general recommendations for the treatment of patients with chronic respiratory failure resulting from bronchiectasis [276]. For the treatment of patients with advanced bronchiectasis, the use of the above-described measures for cystic fibrosis are recommended.

\section{Thoracic-Restrictive Lung Diseases}

\subsection{What Is the General Definition and Disease}

Profile of Thoracic-Restrictive Illnesses?

Chronic respiratory failure in thoracic restrictive diseases is an established indication for HMV $[4,218,255,277$, 278]. Besides severe deformities of the thoracic skeleton, wall, or vertebral column with different underlying aetiologies [34, 279], post-inflammatory or residual post-operative conditions can also lead to this clinical condition (Table 7). An unfavourable breathing mechanism with reduced lung or thoracic compliance can, next to the restrictive ventilation disorder, be seen as an important pathogenetic factor in this disease. The diseases listed in Table 7 generally have a stable or only slow-progressing type of course. An exception to this is pulmonary fibrosis, which frequently takes a rapidly progressing course; this condition is therefore given special attention at the end of this chapter (Ch. 10.6).

\subsection{When Does the Indication for Non-Invasive} Ventilation Exist in Thoracic-Restrictive Disease

\section{Patients?}

The most important criterion for NIV is chronic hypercapnia that is associated with the typical symptoms of respiratory failure or the reduction of quality-of-life. The indication criteria are as follows:

Symptoms of hypoventilation (Ch. 3) and at least 1 of the following observations:

- Chronic diurnal hypercapnia with a $\mathrm{PaCO}_{2}$ of $\geq 45$ $\mathrm{mm} \mathrm{Hg}$

- Nocturnal hypercapnia with a $\mathrm{PaCO}_{2}$ of $\geq 50 \mathrm{~mm} \mathrm{Hg}$

- Normocapnia during the day with a rise in $\mathrm{PtcCO}_{2}$ of $\geq 10 \mathrm{~mm} \mathrm{Hg}$ during the night

Treating Chronic Respiratory Failure with Invasive and Non-Invasive Ventilation
Table 7. Common thoracic-restrictive diseases with an indication for home mechanical ventilation

Kyphoscoliosis

Kyphosis

Pigeon chest

Funnel chest

Bechterew syndrome

Restrictive pleural diseases

Post-tuberculosis syndrome

Post-traumatic thoracic deformities

Post-operative thoracic deformities (thoracoplastic)

Diurnal hypercapnia, however, can actually be preceded by significant nocturnal hypoventilation, first during the REM sleep phase, and also later during non-REM sleep phases [280, 281], which can even worsen the patient's prognosis [282]. Studies on patients either with a VC less than $50 \%$ of that predicted, or with typical symptoms of hypercapnia, have shown that the development of daytime respiratory failure can be favourably influenced by early implementation of NIV when hypoventilation is exclusively nocturnal $[282,283]$. It can be deduced from this observation that the indication for NIV can also be established through nocturnal blood gas or $\mathrm{PtcCO}_{2}$ levels [284]. Patients without manifest hypercapnia, but with severe restrictive ventilatory dysfunction ( $\mathrm{VC}<50 \%$ of the predicted value), should undergo shortterm (within 3 months) clinical monitoring including polygraphy (Fig. 4).

Due to the high prevalence of additional obstructive sleep apnoea, further polygraphic/polysomnographic diagnostics should be aimed for, especially when there is evidence for diurnal normocapnia. As a rule, an intermittent desaturation that takes longer than 5 min under an oxygen saturation level of $<85 \%$ in the polygraphy corresponds to hypoventilation $[285,286]$.

Respiratory insufficiency can also manifest prematurely during pregnancy and in this case serves as an indication for NIV [279]. The indication for NIV initiation can exist in cases of long-term interstitial lung diseases (e.g., before a scheduled lung transplantation) and possibly in the context of rehabilitation [287]; hereby, the individual benefit needs to be examined on a case by case basis. 
Fig. 4. Algorithm for treatment of chronic hypercapnic respiratory insufficiency in patients with thoracic-restrictive diseases. Stepwise approach to implementing NIV therapy in patients with thoracic-restrictive diseases.

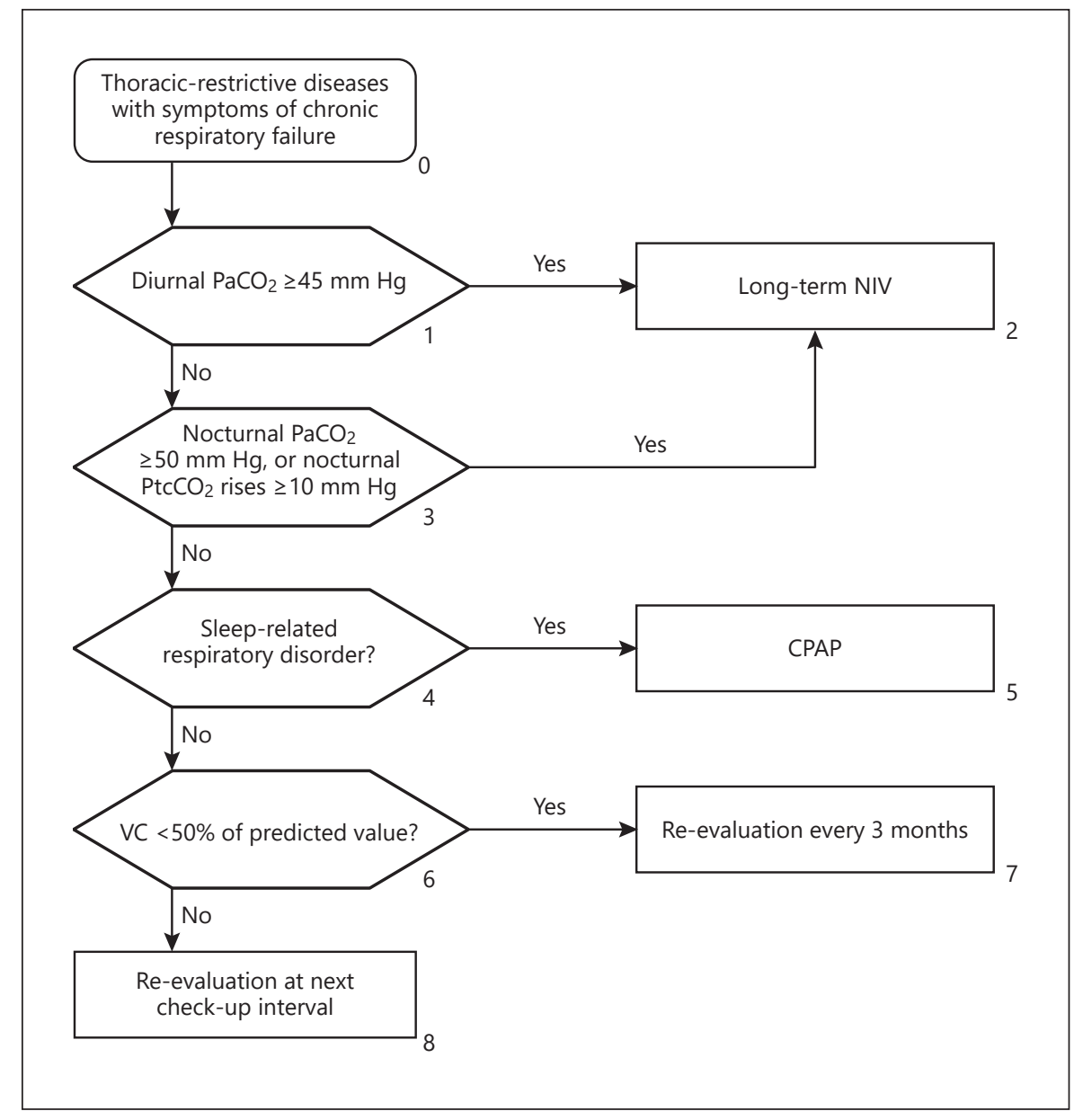

10.3 Which Disease-Specific Aspects of the Ventilation Therapy Should Be Observed in Thoracic-Restrictive Disease?

NIV can take place under pressure-limited as well as volume-limited modes [288-291]. For the pressure-limited ventilation, peak inspiratory ventilation pressures that often exceed 20 mbar are necessary [279]. Switching from pressure to volume presets $[59,289]$, or from volume to pressure presets, may be necessary in individual cases to improve ventilation [292]. PEEP/EPAP is not usually necessary if no further obstruction of the upper airway exists [279].

\subsection{What Are the Proven Effects of Mechanical \\ Ventilation?}

The positive effects of ventilation on thoracic restrictive diseases have been described in various studies [34, 279, 293]. Besides improving typical symptoms (Ch. 5), the positive effects are listed as follows.
Improvements in gas exchange, quality of life, sleep profile, physical resilience, pulmonary haemodynamics, maximal oxygen uptake, lung function, and inspiratory muscle strength, as well as a reduction in the rate of hospitalisation [88, 255, 294-303]. Elderly patients ( $>75$ years) can also benefit from NIV [304]. Furthermore, it can lead to a reduction both in dyspnoea and daytime sleepiness as assessed by the Epworth sleepiness scale [305].

Respiratory muscle unloading [306-308], restoration of central chemosensitivity [309, 310], and the improvement in pulmonary or thoracic compliance with a reduction in restrictive ventilatory dysfunction appear to be the most important underlying mechanisms [302, 303, 311].

In addition, based on several observational studies, it can be assumed that these patients have improved longterm survival under NIV therapy [255, 277, 305]. Studies have shown that in comparison to patients receiving long-term oxygen therapy (LTOT), a clear survival ad- 
vantage exists in patients undergoing NIV, especially in those with severe kyphoscoliosis or post-tuberculosis syndrome $[299,312,313]$.

\subsection{What Particularly Needs to Be Noted for Non-Invasive Ventilation Therapy in \\ Thoracic-Restrictive Disease Patients?}

An interruption to NIV therapy that lasts for a few days can involve a relatively rapid deterioration in blood gases $[283,314]$. LTOT can be additionally necessary for hypoxia that persists despite effective mechanical ventilation [165]. The indication for orthopaedic corrective procedures should be reviewed in cases of thoracic or vertebral column deformities, especially in children and teenagers.

\subsection{What Is the Role of Non-Invasive Ventilation in}

Patients with Pulmonary Fibrosis?

A few studies have described the short-term use of NIV in patients with interstitial lung diseases [287, 315, 316]. Due to the generally poor prognosis, there are no long-term data in relation to NIV in this disease group $[317,318]$. In particular, there are no data for prognosis following the appearance of respiratory failure/hypercapnia; it is, however, assumed that hypercapnia only appears during the end stages of the disease [319]. NIV can be considered here for palliative care purposes. The insecure data situation does not currently allow the formulation of a general recommendation for NIV in pulmonary fibrosis patients [320].

\section{Recommendations}

- Non-invasive ventilation is the primary therapeutic option for home mechanical ventilation of thoracic-restrictive disease patients with chronic respiratory failure. The most important criteria for beginning long-term non-invasive ventilation are hypercapnia in combination with both the typical symptoms of chronic respiratory failure and the reduction in quality of life.

- For symptoms of hypoventilation in the absence of hypercapnia, a sleep study should take place.

- Patients with severe restrictive ventilatory dysfunction but without manifest hypercapnia must be closely monitored.

\section{Obesity Hypoventilation Syndrome}

\subsection{What Is the General Definition of Obesity \\ Hypoventilation Syndrome?}

Next to the ever-increasing worldwide prevalence of obesity, a rising trend towards being overweight has also appeared in Germany. In 2014, the Federal Bureau of Sta-

Treating Chronic Respiratory Failure with Invasive and Non-Invasive Ventilation tistics quoted the increase in the prevalence of obesity within the general population as $15.7 \%$ for men and $13.8 \%$ for women. Obesity prevalence amongst patients who are referred to a sleep lab is estimated to be around $10-20 \%$ [321]. OHS prevalence rises with an increase in the degree of obesity [322]. OHS places higher demands on healthrelated performance and brings about an 8 -fold increase in yearly costs compared to those associated with a healthy control cohort [323], as well as a $15 \%$ higher 5 -year mortality and morbidity rate, even when NIV therapy is applied [321, 324]. Untreated OHS leads to a $23 \%$ increase in mortality [325].

OHS is defined by the presence of obesity (body mass index $[\mathrm{BMI}] \geq 30 \mathrm{~kg} / \mathrm{m}^{2}$ ) in combination with chronic alveolar hypoventilation and subsequent diurnal hypercapnia $\left(\mathrm{PaCO}_{2} \geq 45 \mathrm{~mm} \mathrm{Hg}\right)$ during normal tidal breathing, after ruling out other causes of hypoventilation [326, 327]. A high coincidence exists between sleep-related respiratory disorders (coexistence of OSAS in up to $90 \%$ of cases), which is why sleep medicine diagnostics (via polysomnography) play a decisive role $[326,327]$. Patients with OHS mostly complain of pronounced daytime sleepiness, rapid exhaustion, respiratory distress, or headache. For a number of patients, the disease can also become apparent through signs of right ventricular decompensation, pulmonary hypertension, or polyglobulia $[328,329]$.

Complex pathophysiological mechanisms form the basis of OHS. The extent of diurnal hypercapnia is closely related to BMI, the severity of OSAS, and the magnitude of restrictive ventilation dysfunction [330]. Further important pathogenetic factors are uneconomical breathing mechanisms including extra-thoracic-restrictive ventilatory dysfunction, an increased work of breathing as a result of the commonly occurring obstructive sleep apnoea, as well as extreme obesity in combination with a disruption to central breathing regulation, which manifests as reduced respiratory drive upon hypoxic or hypercapnic stimuli [331-334]. Some studies have provided particular evidence to suggest that the central respiratory-stimulating effect of leptin is reduced in OHS [335-337]. However, like COPD, OHS is not to be treated purely as a pulmonary disease; instead, all the systemic effects of this condition with respect to the accompanying cardiovascular and metabolic symptoms should be addressed in the therapy plan $[321,338]$.

\subsection{What Are the Indications for the Initiation of} Home Mechanical Ventilation Therapy in Obesity Hypoventilation Syndrome Patients?

Patients with OHS almost always exhibit OSAS, which plays a central pathogenic role. Therefore, a primary sleep 
diagnostic test using polysomnography is essential [ 327 , 339-341]. Simplification of the OHS diagnostic process by using evidence for increased bicarbonate levels in the blood $\left(\mathrm{HCO}_{3}>27 \mathrm{mmol} / \mathrm{L}\right)$ or base excess $(>3 \mathrm{mmol} / \mathrm{L})$ through a daytime blood gas analysis is currently being discussed for patients presenting with obesity and its corresponding clinical symptoms; however, this cannot be recommended as a single diagnostic criterion [340, 342, 343].

In most cases, CPAP therapy is necessary, and this should be titrated under both poly(somno)graphic surveillance and nocturnal measurements of $\mathrm{PCO}_{2}$, to the point where nocturnal desaturations are sufficiently controlled $\left(\mathrm{SpO}_{2}>90 \%\right)[25,327,341,344,345]$. In general, a regular CPAP device normally used for sleep apnoea therapy can be used for CPAP therapy in OHS patients [341]. In the event that nocturnal hypopnoea, arousal reactions through increased work of breathing (respiratory effort-related arousal, RERA), and hypoventilation can all be either eliminated or significantly reduced under CPAP therapy, then this therapy should be continued [327, 340, 344].

If a long-standing drop in $\mathrm{SpO}_{2}(<80 \%)$ and/or a clinically relevant rise in $\mathrm{PCO}_{2}$ take(s) place, the patient should be switched from CPAP to NIV. Piper et al. [344] reported a clinically relevant rise in $\mathrm{PCO}_{2}$ that was defined either by an increase in $\mathrm{PtcCO}_{2}$ of $\geq 10 \mathrm{~mm} \mathrm{Hg}$ during REM sleep or an increase in diurnal $\mathrm{PaCO}_{2}$ of $\geq 10$ $\mathrm{mm} \mathrm{Hg}$ in patients with a starting $\mathrm{PaCO}_{2}$ of $>55 \mathrm{~mm} \mathrm{Hg}$. The indication for switching from CPAP to NIV arises either from CPAP intolerance, or from one of the following situations under fully titrated CPAP therapy:

- Clinically relevant increase in nocturnal $\mathrm{PCO}_{2}$

- Persistent hypopnoea and RERA

- Prolonged periods ( $\geq 10 \mathrm{~min})$ of desaturation $\left(\mathrm{SpO}_{2}\right.$ $<80 \%)$

Regarding sleep-related respiratory dysfunction (hypopnoea/RERA), explicit reference should be made to the DGSM S3 Guideline for unrestful sleep. It is worth noting here that no evidence-based cut-off value exists for either hypopnoea or RERA, making it necessary to consider the manifestation of nocturnal respiratory dysfunction on an individual basis [341].

If there is no improvement in the typical symptoms of chronic hypoventilation after 3 months of CPAP therapy, and diurnal normocapnia cannot be detected during patient re-evaluation ("non-responder"), a switch to NIV is indicated [344] (Fig. 5). Set-up and monitoring of CPAP and NIV should take place ideally under polysomnography or alternatively under polygraphy evaluation [341].
Notwithstanding this approach, NIV can also be indicated if pronounced hypercapnia is initially evident or severe symptomatic comorbidities such as accompanying cardiovascular diseases are present [338], since the adjustment of $\mathrm{PCO}_{2}$ is more easily achieved under NIV than under CPAP therapy [346]. Given the combination of the above-described complexities, the systemic nature of OHS, and the initiation of therapy, the need for extended in-hospital treatment often exists. Based on currently available data, the suggested approach to therapy initiation is summarised in a clinical flowchart (Fig. 5).

\subsection{What Are the Disease-Specific Features}

of Mechanical Ventilation Therapy in Obesity

Hypoventilation Syndrome?

The fundamental treatment objectives are to eliminate hypoventilation and hypercapnia, and provide therapy for the sleep-related respiratory diseases that are most commonly associated with OHS. According to the abovementioned scheme, the response to the initial CPAP therapy determines the selection of the positive-pressure method (CPAP or NIV).

Patients with a combination of OHS and OSAS who have been introduced to NIV therapy should be subjected to sufficiently high PEEP/EPAP levels in order to offset obstructions of the upper airways [339]. Patients who are first diagnosed with OHS in the course of acute hypercapnic failure and then treated accordingly with NIV therapy may continue under this therapy regimen [347]. Depending on whether clinically relevant weight loss occurs, an attempt at switching to CPAP therapy under poly(somno) graphic evaluation with an adjoining re-evaluation of sleep at 3 months can be discussed once NIV has been established (Fig. 5). There is, however, a lack of scientific evidence to support a general recommendation in this context [348]. Nevertheless, a recent study showed that irrespective of weight loss, a de-escalation of therapy from NIV to CPAP can be possible without loss of the associated therapeutic effects; hence, this option is often most favoured by patients. This should be evaluated accordingly with polysomnography in consultation with the patient during stable phases of the disease [349].

\subsection{What Kind of Effects of Mechanical Ventilation}

Can Be Expected Based on Research Findings?

The use of NIV has proven to be an effective therapeutic option [350]. Most studies dealing with the long-term effects of NIV in patients with OHS have found an improvement in blood gases during both nocturnal mechanical ventilation and diurnal spontaneous breathing, 


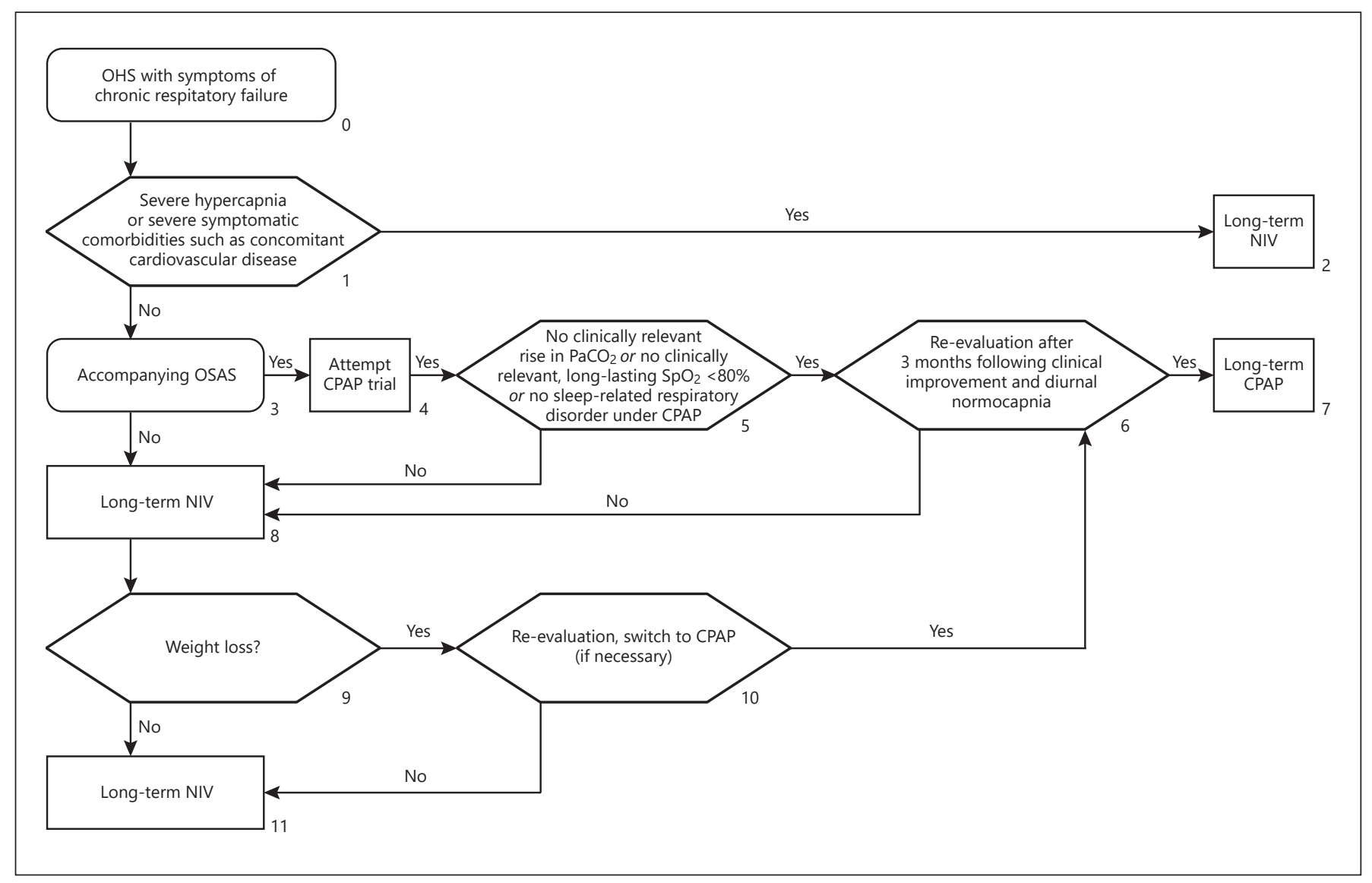

Fig. 5. Algorithm for treatment of chronic hypercapnic respiratory insufficiency in patients with OHS. Stepwise approach to NIV therapy in patients with OHS.

a reduction in daytime sleepiness and dyspnoea, and improved sleep quality and quality of life [327, 329, 339]. In addition, following the initiation of NIV with higher ventilation pressures, positive effects on static lung function parameters were found that are mainly based on an increased expiratory reserve volume [351-353]. Physiological studies suggest that high-intensity NIV also leads to respiratory muscle unloading [354].

Recent studies have investigated the effects of the socalled hybrid modes of NIV in patients with OHS. Although it was initially presumed that hybrid modes provide the advantage of improved ventilation, this could not be confirmed in subsequent studies. This means that these modes may still be used, but do not offer any significant advantages over conventional NIV [65, 68, 355]. Comparative retrospective analyses have shown that OHS patients who are not treated with NIV have a significantly higher mortality rate than those who do undergo NIV therapy $[325,350,351,356]$.

Treating Chronic Respiratory Failure with Invasive and Non-Invasive Ventilation
11.5 What Are the Additional Therapy Options for Obesity Hypoventilation Syndrome Patients?

Blood gas improvement in terms of reduced hypercapnia and hypoxemia is closely related to the duration of NIV use. One particular study reported positive effects after a treatment duration of at least 5-7 h/night [357]. Invasive ventilation should be considered as a last resort for progressive respiratory insufficiency with respiratory acidosis [358]. In untreated OHS, oxygen application can lead to hypoventilation with respiratory acidosis; this therefore requires close monitoring of gas exchange and, if necessary, NIV use in the event of acute respiratory failure [359].

In addition, marked weight loss can contribute to a significant reduction in chronic respiratory failure [360]. However, in the majority of OHS patients, clinically significant weight loss is not achieved in the long term through dietetic measures alone [351, 352]. Surgical intervention options such as bariatric surgery can addition- 
ally be evaluated; further details on this topic can be found in guidelines from the relevant professional societies [361]. In clinically suspected OHS, appropriate diagnostic tests for OHS or nocturnal respiratory dysfunction are recommended before operative measures.

\section{Recommendations}

- Continuous positive airway pressure or non-invasive ventilation are the primary treatment options for home mechanical ventilation in patients with obesity hypoventilation syndrome.

- A primary attempt at continuous positive airway pressure therapy must take place under poly(somno)graphic evaluation. Primary non-invasive ventilation can be indicated in the presence of significant comorbidities or severe hypercapnia.

- A switch to non-invasive ventilation is indicated in the following cases: continuous positive airway pressure intolerance, persistent sleep-related or aggravated hypoventilation, and/or persistent sleep-related respiratory dysfunction despite continuous positive airway pressure use.

- Concomitant weight loss should be aimed for.

- A therapeutic switch from non-invasive ventilation to continuous positive airway pressure should be considered under stable conditions, particularly after weight reduction; if applicable, omission of mechanical ventilation can be considered.

- If obesity hypoventilation syndrome is clinically suspected, appropriate diagnostic tests for obesity hypoventilation syndrome should take place before undergoing general anaesthetics.

\section{Neuromuscular Diseases}

\subsection{Which Types of Neuromuscular Diseases Lead to Respiratory Failure?}

The extent and frequency of respiratory muscle involvement varies amongst different NMDs. The extent of respiratory muscle paralysis often correlates with that of general muscle weakness and physical disability, although there are exceptions: in 10\% of patients with amyotrophic lateral sclerosis (ALS), early-stage respiratory muscle weakness can be demonstrated, not uncommonly as the first symptom. This also holds true for the adult form of glycogenosis type II (lack of alpha-glucosidase, Pompe disease) and some subforms of limb-girdle muscular dystrophy. Diseases that can involve sleep-related respiratory dysfunction and/or chronic respiratory failure are summarised in Table 8.

Physical capacity per se is progressively reduced due to general muscle weakness. Therefore, muscular respiratory insufficiency (respiratory pump weakness) in NMD patients rarely shows early clinical manifestation in the form of exercise-induced dyspnoea.
In general, respiratory failure primarily manifests as hypoventilation during sleep. A forced vital capacity (FVC) $<70 \%$ can lead to REM-sleep-associated hypoventilation; an FVC $\leq 50 \%$ commonly causes hypoventilation in other stages of sleep. Typical corresponding diurnal symptoms manifest at the latest when FVC reaches $<20$ $40 \%$ [362]. In this light, nocturnal polygraphic or polysomnographic testing that includes $\mathrm{PtcCO}_{2}$ measurement is of particular importance for patients with NMD.

\subsection{What Are the Indications for Home Mechanical}

Ventilation in Neuromuscular Diseases Patients?

12.2.1. What Sort of Screening Tests (Especially for

Respiratory Pump Weakness) Are Available, and

When and How Often Should They Be Performed?

If a patient presents with an NMD that is associated with the risk of developing respiratory pump weakness, regular screening (every 3-12 months, depending on clinical course and type of NMD) of lung function and blood gases should be performed, rather than first during respiratory decompensation. Evaluation includes poly(somno)graphy and optional $\mathrm{PtcCO}_{2}$ testing when FVC is $<70 \%$ for timely detection of respiratory pump weakness. Polygraphy can in certain situations (presence of severe OSAS) and in some diseases (e.g., patients with myotonic dystrophy type 1, Curschmann-Steinert syndrome) be inadequate for diagnostic testing and should be supplemented by polysomnography [363]. The tests listed in Chapter 5 include obligatory testing of coughing capacity. Many genetic forms of NMD are associated with clinically relevant cardiac involvement. To prevent cardiac involvement in the form of conduction impairment and/or dilated cardiomyopathy from being overlooked, regular ECG, Holter-ECG, and echocardiographic monitoring should be performed at both early and later stages of the disease. These cardiac diagnostic tests are especially important since cardiomyopathy itself can often be associated with forms of central apnoea and/or CheyneStokes respiration.

12.2.1.1 What Is the Significance of Polysomnography? Polysomnography in combination with $\mathrm{PtcCO}_{2}$ measurement and blood gas analysis in the morning can be useful for early diagnosis of nocturnal hypercapnia. The exact classification of REM-associated phases of hypercapnia can only be achieved by polysomnographic sleep examination. A further reason for performing polysomnography in NMD patients is the common presence of OSAS due to pharyngeal muscle weakness, which can precede the development of respiratory failure by months to years. This particularly holds true for ALS patients. In addition, 


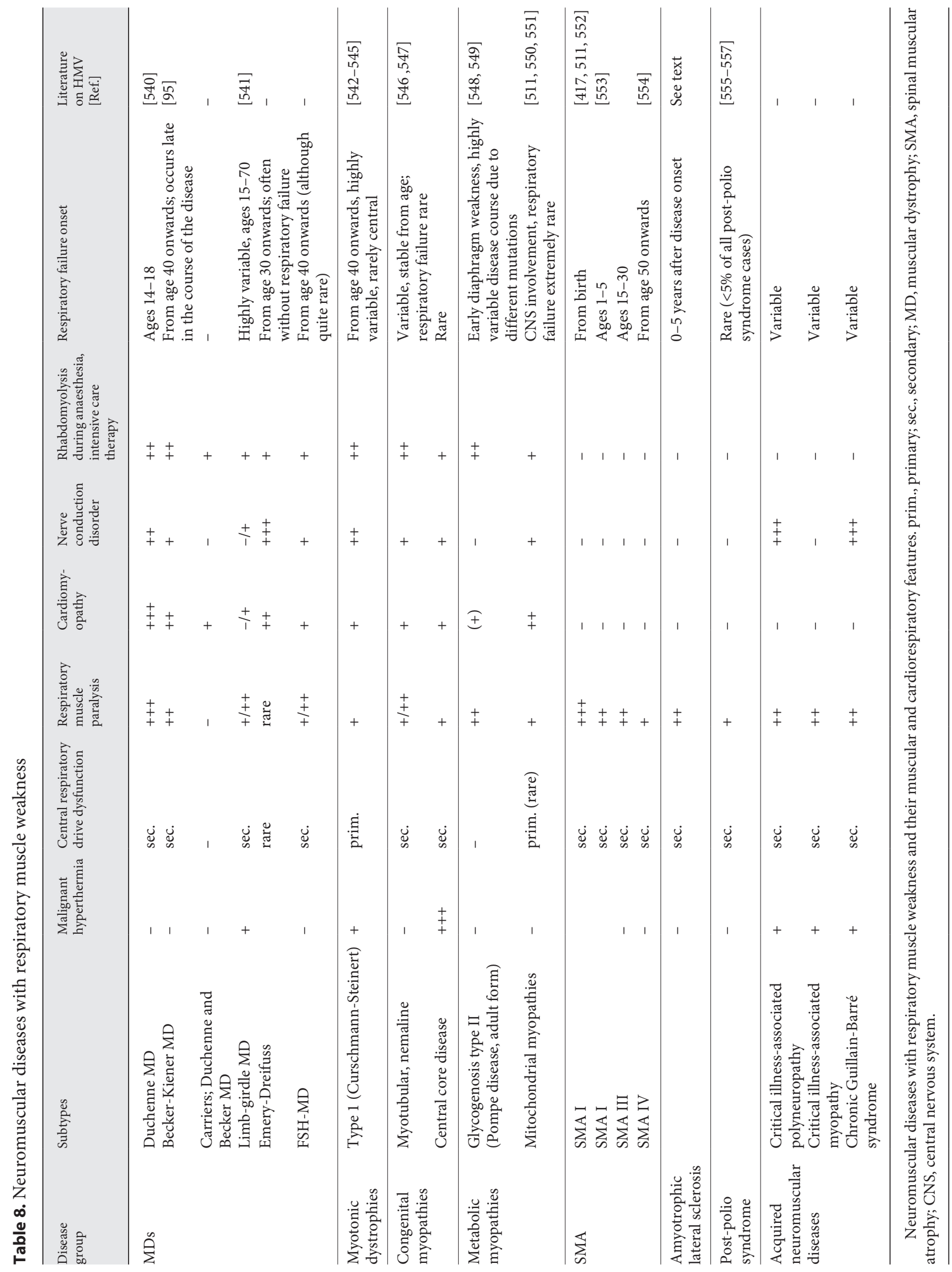




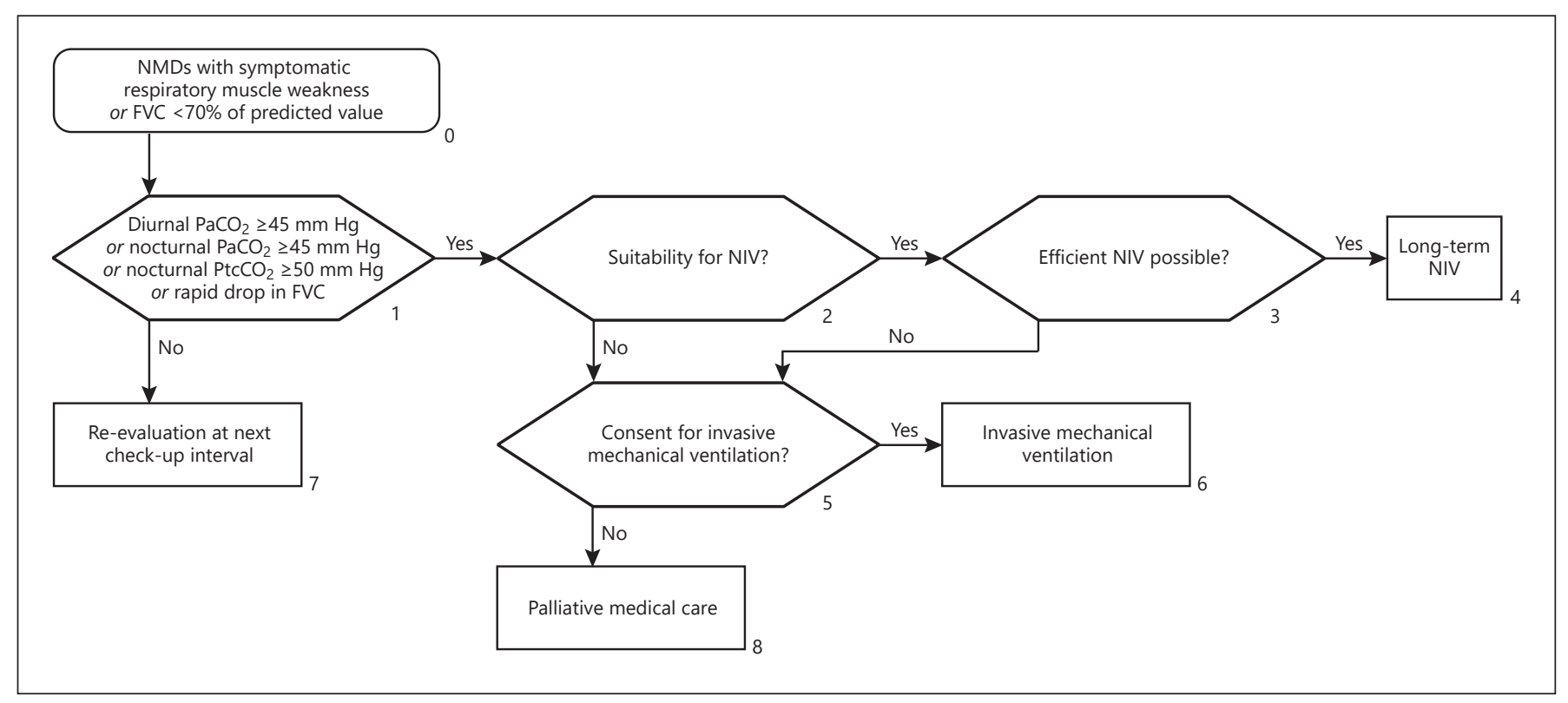

Fig. 6. Algorithm for treatment of chronic hypercapnic respiratory insufficiency in patients with NMD. Stepwise approach to establishing an indication for mechanical ventilation in patients with NMD.

early detection of central apnoeas - especially in Curschmann-Steinert patients - is possible with this approach. Similarly, the use of polysomnography during the initial set-up of NIV provides clearer information about the improvement of sleep quality and allows reliable allocation of the sleep phases to the corresponding saturation and capnometry data [364-367]. $\mathrm{PtcCO}_{2}$ measurement is the key technique for this diagnosis and is indispensable for therapy initiation and monitoring [368].

12.2.1.2 When Does the Indication for Non-Invasive Ventilation Arise in Neuromuscular Disease Patients?

The indication for NIV in NMD patients [277, 279, 369-379] comprises the presence of hypoventilation symptoms and at least one of the following results (Fig. 6):

- Diurnal $\mathrm{PaCO}_{2} \geq 45 \mathrm{~mm} \mathrm{Hg}$

- Nocturnal $\mathrm{PaCO}_{2} \geq 45 \mathrm{~mm} \mathrm{Hg}$

- Nocturnal $\mathrm{PtcCO}_{2} \geq 50 \mathrm{~mm} \mathrm{Hg}$ for over $30 \mathrm{~min}$

- Diurnal normocapnia with a nocturnal rise in $\mathrm{PtcCO}_{2}$ of $\geq 10 \mathrm{~mm} \mathrm{Hg}$

- For ALS or other fast-progressing NMDs, a rapid reduction in FVC of $>10 \%$ of the initial value within 3 months

Nocturnal hypoventilation is also indicative of nocturnal desaturation. As a consequence, long-lasting nocturnal desaturation (intermediate $\mathrm{SpO}_{2}<90 \%$ or $\mathrm{SpO}_{2}<90 \%$ for at least $10 \%$ of the time period measured) can also hint at hypoventilation that is clinically relevant. Nocturnal desaturations alone cannot, however, serve as an indication for NIV initiation, since other causes of desaturation (OSAS, pulmonary insufficiency, secretion build-up) also need to be taken into account.

ALS can be distinguished from most other NMDs by its fast disease progression. The average life expectancy without mechanical ventilation is $2-5$ years from disease onset, whereby $>10 \%$ of patients die as early as within the first year from consequences of respiratory muscle weakness, if this is not treated [380]. When FVC is $<70 \%$ and there is a reduction in FVC of $>10 \%$ in 3 months, nocturnal NIV can be started regardless of further test results, due to the rapid course of the disease. Positive effects on subjective and quantified (polysomnography) sleep quality have been demonstrated in ALS patients [381]. Improvements in sleep parameters and diurnal symptoms have also been shown for other NMDs [382]. NIV quality, implementation, and support are, however, the decisive factors for the subjective improvement of sleep in NMD patients [383].

There is no indication for prophylactic mechanical ventilation therapy when diurnal clinical symptoms and hypoventilation are lacking; rather, based on a study in Duchenne muscular dystrophy patients, there are actually concerns that this can lead to a deterioration in prognosis [384]. An exception to this occurs prior to planned spinal correction surgery when $\mathrm{VC}$ is $<60 \%$ of the target 
value, or FEV1 is $<40 \%$ [385]: in this case, the frequency of post-operative complications can be reduced through pre-operative initiation of prophylactic HMV.

NIV can be considered in isolated cases, such as during pregnancy [386], and with already restricted lung function, or for palliation of dyspnoea at end stages of the disease [387]. If diagnostic uncertainties arise, an attempt at NIV therapy can be carried out. If symptoms are ameliorated under NIV (therapy success), the requirement for therapy is validated.

Instead of waiting for diurnal hypercapnia to develop the patient should be offered NIV upon the first signs of nocturnal hypercapnia (e.g., increase in bicarbonate). One study was able to show that in many patients with primary nocturnal hypercapnia, deterioration takes place within a time frame of 2 years, with development of diurnal hypercapnia as well as acute respiratory decompensation [282]. Disease progression should be taken into account during the indication process: indication should be generously made in fast-progressing diseases such as ALS, since an improvement in diurnal symptoms can be expected. In some patients, 24-h NIV without optional spontaneous breathing is possible [388], but places high demands on their care and surveillance. The decision to implement ongoing invasive mechanical ventilation should be made independent of this, because sufficient support can indeed lead to a quality-of-life level that is comparable to that under NIV [389-391].

Patients with slowly progressing weakness of the respiratory pump can also develop clinical signs through initial nocturnal hypoventilation. This can be detected with high sensitivity through nocturnal $\mathrm{PtcCO}_{2}$ measurements [368].

12.2.1.3 When Should Phrenic Nerve Stimulation and Direct Diaphragm Pacing Be Considered?

The use of electrical stimulation methods to improve breathing is linked to neuromuscular integrity (lower motoneuron - neuromuscular transmission - muscle). While this is successfully applied in patients with spinal cord transection above $\mathrm{C} 3$, it is not effective in NMD that affects upper and lower motoneurons, the phrenic nerve, neuromuscular transmission, or the muscle per se. This approach is associated with shortened survival and is therefore obsolete [392].

\subsubsection{When Does the Indication for Invasive}

Mechanical Ventilation Arise in Neuromuscular

Disease Patients?

Mechanical ventilation via tracheostoma in NMD patients is possible over a duration of many years. The un- derlying disease itself (e.g., cardiomyopathy in Duchenne muscular dystrophy) and ethical concerns (complete loss of voluntary function in ALS patients within 2-5 years) are the limiting factors. The patient and his/her family should be fully informed about the course of the disease prior to the decision being made; consent from the patient or legal guardian is mandatory to prevent intubation and tracheotomy, which are not uncommonly carried out against the patient's will in emergency situations. On the other hand, recent data show that decent quality of life can by all means be achieved in invasively ventilated patients with NMD [391]. This also pertains to isolated cases of 24-h NIV.

Upon the patient's wish and consent, tracheotomy is indicated for the following situations $[369,376,388,393$, 394]:

- Inability to fit an appropriate ventilation interface

- NIV intolerance

- NIV inefficiency

- Severe bulbar symptoms with recurrent aspiration

- Inefficiency of non-invasive secretion management

- Failure to switch to NIV after intubation and invasive ventilation

\subsection{Which Disease-Specific Aspects of the}

Mechanical Ventilation Therapy Should Be Noted for

Neuromuscular Disease Patients?

There are 3 special features of NMD patients that are critical for choosing the ventilation interface, deciding between NIV or invasive ventilation, and selecting the accompanying therapy for secretion management:

- Oropharyngeal muscular weakness with the risk of mouth closure inability/insufficiency

- Bulbar symptoms with the risk of recurrent aspirations or sialorrhea

- Coughing weakness with inadequate secretion clearance that is not compensated by assisted-coughing techniques

\subsubsection{Muscle Weakness}

When oropharyngeal muscles are affected, mouth closure can be impaired to the point where effective ventilation via a nasal mask is no longer possible. As an alternative, the use of an oronasal mask may be necessary. A chin band in combination with an oronasal mask can be a burden for the patient and is therefore only rarely put into use. In some cases, pronounced weakness of the facial muscles can represent an indication for tracheotomy. 


\subsubsection{Bulbar Symptoms}

Aspiration with concomitant coughing weakness is particularly associated with the risk of high airway secretion loading and infections. In mild cases, intensive secretion management therapy (see below) can be successful; in severe cases, the prognosis is highly compromised without tracheotomy [395]. In ALS patients, the presence of marked bulbar symptoms as an indicator for NIV initiation success is controversial [372, 374, 377, 396-399]. An attempt at NIV therapy is recommended in any case, as long as the patient tolerates mechanical ventilation and benefits in terms of diurnal symptoms [400, 401]. Sialorrhea, which affects breathing in patients with bulbar ALS, is the result of dysphagia (so-called pseudo-hypersalivation). Saliva formation can be reduced by the application of anticholinergic substances (scopolamine TTS among others); because of its concomitant anti-depressive and sleep-triggering effect, the anti-depressant amitriptyline can primarily be used. Injections of botulinum toxin type A into the salivary glands also reduce saliva formation and can be applied by experienced operators at 2- to 6 -month intervals $[402,403]$. In cases of untreatable sialorrhea with severe aspiration tendency, tracheotomy to prevent aspiration can be indicated even without ventilation, as long as the patient consents to this after extensive clarification.

\subsubsection{Weak Cough}

A weak cough can be contingent on individual or combined weakness of the inspiratory, expiratory, or glottis muscles. Early diagnostic tests comprising anamnestic details, peak cough flow (PCF) measurement and the implementation of an individualised secretion management program (see below) if PCF $<270 \mathrm{~L} / \mathrm{min}$ can help prevent acute decompensation. The subject of cough assistance and cough support is presented in detail in Chapter 13 $[404,405]$.

\subsection{What Sort of Effects Can Be Achieved Through Mechanical Ventilation in Neuromuscular Disease Patients?}

The following benefits of NIV in NMD patients have been demonstrated in a number of uncontrolled studies as well as one controlled study [398]:

- Improvement in blood gases [96, 406]

- Resetting of the respiratory centre with an increase in the hypercapnic-ventilatory response [38, 309]

- Increase in pulmonary compliance $[407,408]$

- Improved sleep quality [112, 381, 383, 397, 406, 409411]
- Improvement in health-related quality of life [409, 411-415]

- Regression of hypoventilation symptoms [406]

- Improvement of depression [409]

- Reduction in the rate of rehospitalisation $[108,112$, 304, 416]

- Prolonged survival [96, 372, 374, 377, 395, 396, 398, 409, 416-421]

- Improvement in neurocognition [414, 422]

\subsection{What Should Be Observed during the Transition}

from Childhood/Adolescence to Adulthood in Patients with Neuromuscular Disease?

For young patients, the step towards becoming an adult also represents a transition from paternalistic-oriented paediatrics to adult medicine, in which the self-initiative of the patient is called upon. This transition should be a smooth process, and the transfer of responsibility from the paediatrician to the ongoing treating physician should ideally take place in a HMV centre [423].

Details about medical history, significant findings (genetics, biopsy results), social-medical aspects, and parameters for respiratory physiology and ventilation should be handed over in a structured manner. The endeavours of the German Society of Transitional Medicine as well as relevant publications serve as a reference for this process [424]. Some forms of NMD lead to clinically relevant respiratory insufficiency in childhood; these include $\mathrm{Du}$ chenne muscular dystrophy, the childhood progressive form of spinal muscular dystrophy, congenital myopathies, and other forms of hereditary neuropathies. In addition to the particular aspects of NIV in paediatrics, the special features associated with the transition from paediatric/adolescent medicine into adulthood should be noted [425]. The subject of transitioning into adult medicine should be discussed with adolescents and their families as early as possible [426]. It is also necessary to establish contact in a timely manner with the HMV centre that will take on the future adult patient. In addition to the continuation of ventilation therapy and the establishment of a connection with a centre specialising in the ventilation of NMD patients, interdisciplinary care from internists and neurologists, as well as paediatricians in the transitional phase, should take place [427]. The transition process should also take into account the individual care and supervision circumstances, as well as the severity and course of the particular NMD [428].

In the event of sustained critical illness polyneuropathy/-myopathy (CIP/CIM) with an indication for HMV, reference to the S2k Guideline "Prolonged Weaning" is 
particularly important, since - unlike other neuromuscular diseases - potential amelioration of muscle weakness with secondary successful weaning/decannulation can exist, even after a period of months [17].

\section{Recommendations}

- Clinical assessment and determination of forced vital capacity in neuromuscular disease patients should take place at 3to 12 -month intervals. A forced vital capacity $<70 \%$ is an indicator for poly(somno)graphy and $\mathrm{PtcCO}_{2}$ measurement.

- In the event of mild hypercapnia $\left(\mathrm{PaCO}_{2} \geq 45 \mathrm{~mm} \mathrm{Hg}\right.$ or diurnal normocapnia with a nocturnal rise in $\mathrm{PtcCO}_{2}$ of $\geq 10$ $\mathrm{mm} \mathrm{Hg}$ ), non-invasive ventilation should be implemented as the primary therapy option for home mechanical ventilation in neuromuscular disease patients with chronic respiratory failure, if diurnal symptoms persist.

- There is no indication for prophylactic implementation of non-invasive ventilation in the absence of chronic respiratory failure.

- The measurement of coughing capacity is obligatory in neuromuscular disease patients. The presence of weak cough (peak cough flow $<270 \mathrm{~L} / \mathrm{min}$ ) is an indication for the introduction of secretion management.

\section{Secretion Management}

\subsection{What Is the Physiology of Bronchial Secretion?}

The submucosal glands and goblet cells of the airways normally produce $<10 \mathrm{~mL}$ of bronchial secretions per day [429]. The bronchial secretion is composed of 2 layers: a thin fluid layer on the cellular side (sol layer) and a sticky, viscous layer on the luminal side (gel layer), which facilitate the binding of inhaled foreign bodies, dust, bacteria, and viruses. Secretion removal takes place via orderly ciliary movements in the ciliated epithelium, with a beating frequency of around $12 \mathrm{~Hz}$; this results in mucous transport towards the larynx. Coughing serves as an alternative mechanism to secretion clearance in cases of damage to, or overexertion of, mucociliary clearance. The interaction of many factors is essential for an effective cough: sufficiently deep inhalation to bring air behind the secretion, glottis closure for simultaneous tension of the expiratory muscle with intrathoracic pressure increase, and subsequent active dilatation of the glottis under continuous tension of the expiratory muscles to achieve a high, outwardly directed current in the airways. Reduction in tracheal diameter via protrusion of the pars membranacea, and the physical feature of thixotropy of the secretion, also play a role. The maximum air discharge velocity during coughing is described as PCF.

The normal values for PCF lie above $360 \mathrm{~L} / \mathrm{min}$, a routine measurement of PCF should be performed at regular
Table 9. Diseases with disrupted secretion clearance that may require assistance in secretion clearance

Obstructive airway diseases

Bronchial asthma

COPD

Cystic fibrosis

Bronchiectasis

Primary ciliary dysfunction

Attenuated or lacking cough

Neuromuscular diseases

Spinal cord paralysis

Intensive care unit-acquired muscle weakness

Recurrent aspiration

Presence of an invasive ventilation interface (tracheal cannulae)

intervals in NMD patients. For values $<250-270 \mathrm{~L} / \mathrm{min}$, the risk of coughing capacity decompensation exists in the event of an infection [430]. According to a study in ALS patients, a considerably restricted cough (PCF $<160$ $\mathrm{L} / \mathrm{min})[431]$ and persistent hypoxemia $\left(\mathrm{SpO}_{2}<95 \%\right)$ are associated with a high risk of dying or needing a tracheotomy, that is if the non-invasive measures for improving secretion expectoration (see below) are not effective [388]. Many illnesses are accompanied by an increase in bronchial secretions and/or a reduction in secretion clearance (Table 9).

Of particular relevance to HMV are, on one hand, those patients with invasive mechanical ventilation, through which the normal mechanisms of secretion clearance (mucociliary clearance and cough) are restricted. On the other hand, secretion management for diseases such as chronic obstructive airway diseases (COPD, cystic fibrosis, and bronchiectasis) or diseases with diminished cough such as a number of NMDs or spinal cord transection cases above the level of T 10 (e.g., paralysis of the expiratory musculature) also plays a role. The composition of the bronchial secretion can also vary, depending on the underlying disease; e.g., a higher DNA secretion content derived from dead bacteria is responsible for the high viscosity of secretions in cystic fibrosis [432].

With regard to subglottic suctioning via a tracheal cannula in invasive mechanical ventilation, the Commission for Hospital Hygiene and Infection Prevention (German: KRINKO) at the Robert Koch Institute recommends the use of endotracheal tubes with means for subglottic suctioning when the expected ventilation duration is $>72 \mathrm{~h}$ [433]. A meta-analysis of 17 studies covering a total of 3,369 patients confirmed this effect, albeit without being able to show the repercussions for mechanical ventilation 


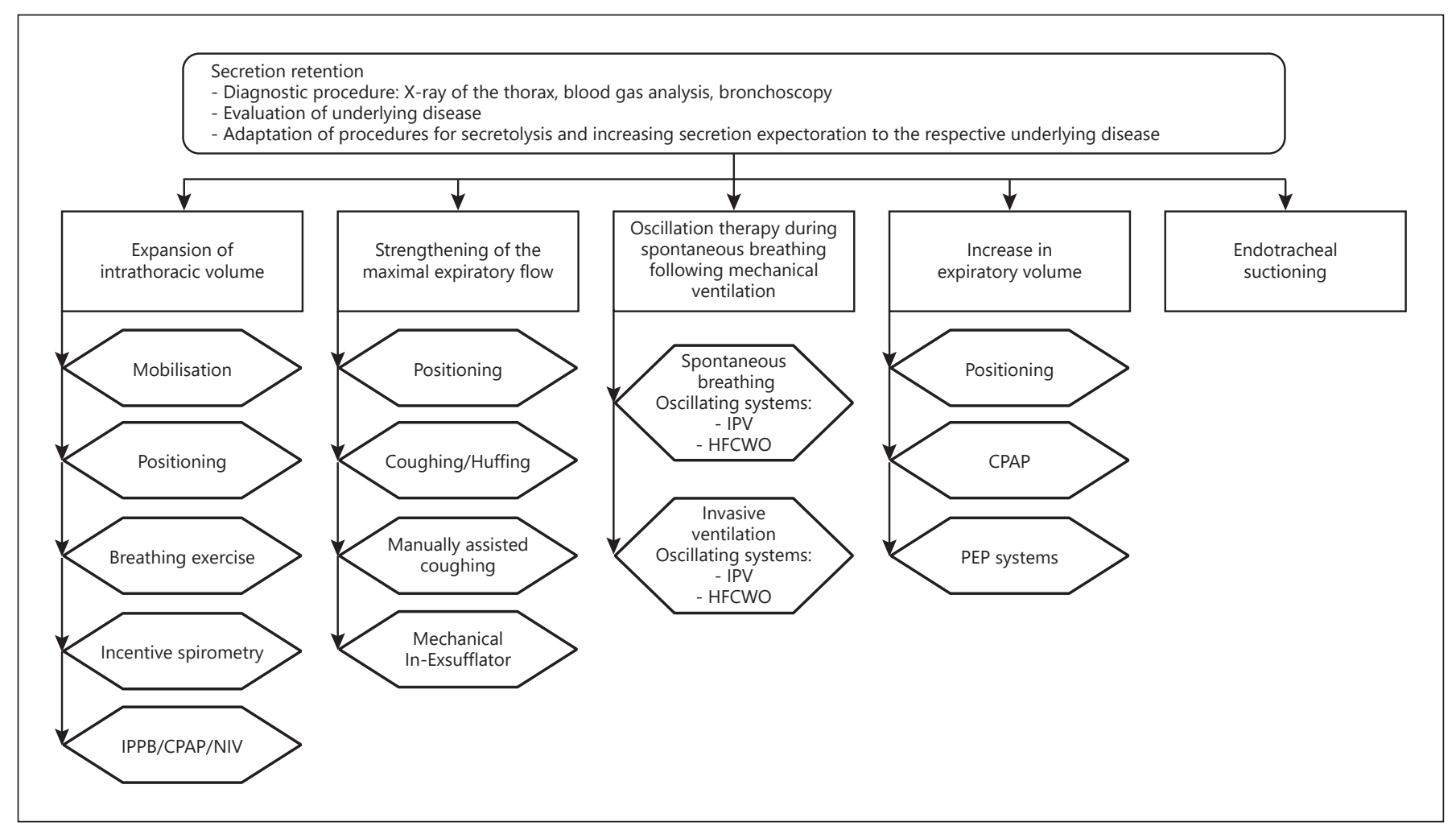

Fig. 7. Measures for improving secretion clearance according to [17]. HFCWO, high-frequency chest wall oscillation; PEP, positive expiratory pressure; IPV, intrapulmonary percussive ventilation.

duration, length of intensive care unit and hospital stays, and mortality rate [434]. This would mean by analogy that only tracheal cannulae with a subglottic suction option should be implemented in invasive HMV. However, such a recommendation is not given in the current guideline for the following reasons:

- There are no existing data on microbiological colonisation in invasive HMV patients or on the prevalence of pneumonia.

- Neither the necessary measures for the prevention of ventilation-associated pneumonia in the outpatient sector nor their effectiveness have been investigated yet.

- Only a few cannula models are equipped with the option for subglottic suctioning. The different anatomy of each patient requires individualised tracheal cannula selection in order to identify an optimally positioned cannula and, in turn, prevent long-term tracheal complications such as granulation tissue or bleeding with endotracheal suctioning. For this reason, many patients cannot be supplied with a cannula fitted with a subglottic suctioning option.

\subsection{What Are the Consequences of Reduced Secretion} Clearance for Home Mechanical Ventilation?

Secretion shifting in the airways can be responsible for NIV failure or the deterioration of the ventilation situation in invasive HMV patients. Besides the increase in ventilation-perfusion inhomogeneities with resulting hypoxemia, an increase in the work of breathing can arise through narrowing of the cross-sectional airways. The risk of infection also increases with clinically relevant secretion retention.

\subsection{How Can Secretion Clearance Be Improved?}

An improvement in secretion clearance can take place through 2 different mechanisms: measures for secretion lysis or mobilisation and measures for improving secretion removal from the airways. From a physiological perspective, there are 5 principles that can be applied for the improvement of secretion mobilisation and removal (Fig. 7).

Distinguishing between the two different aspects of secretion management is of fundamental importance for diverse application of the measures listed in Table 10. 
Table 10. Measures for improving secretolysis or secretion expectoration

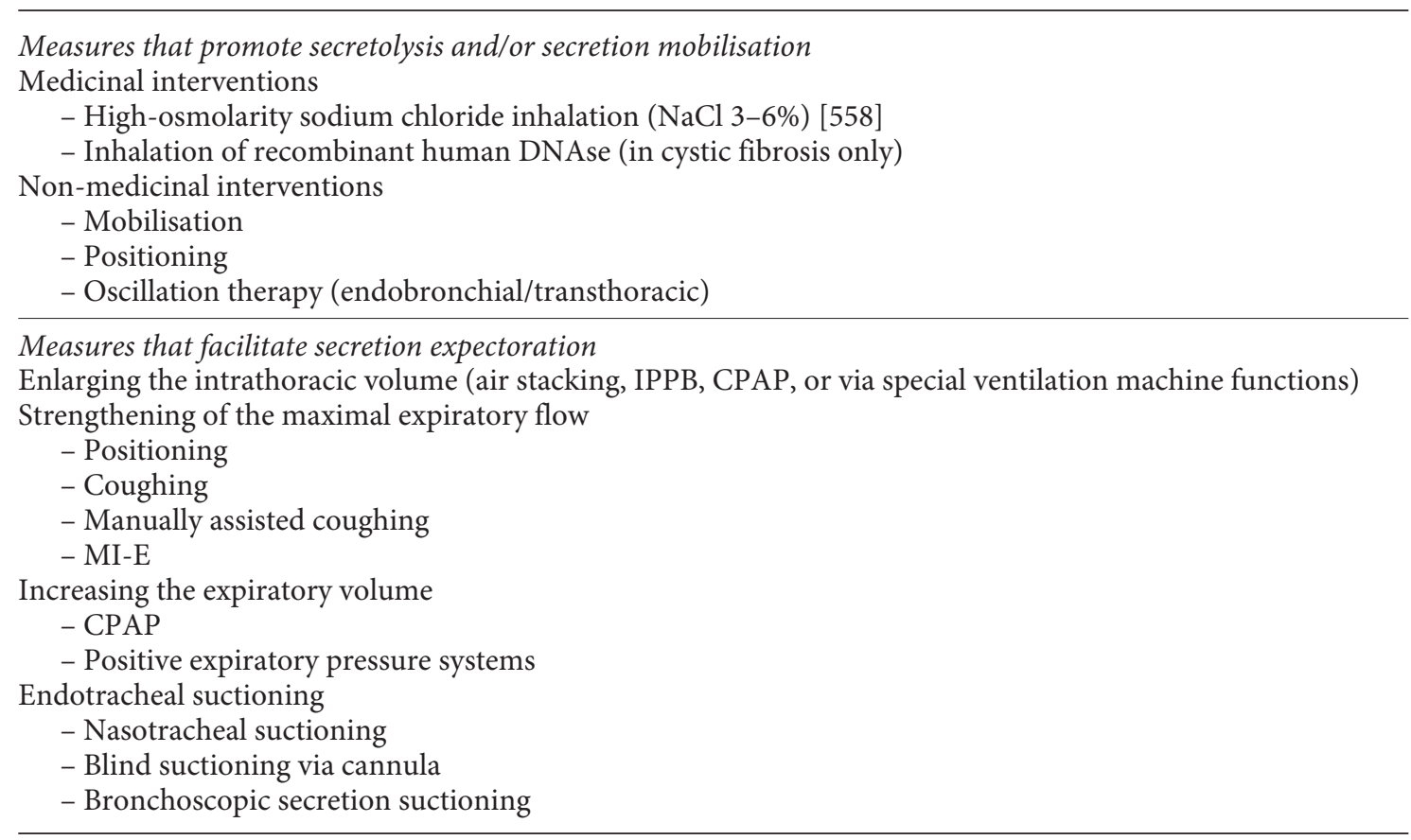

Measures for secretolysis such as inhalation and oscillation therapies can also be used in combination, particularly in invasively ventilated patients. Endotracheal suctioning should always be ensured and performed following secretolysis therapy in invasively ventilated patients. A detailed description of secretion management is beyond the scope of this Guideline. Therefore, only specific problems, especially those arising in NMD patients and invasively ventilated COPD patients, will be discussed from hereon.

Besides the known advantages, the different techniques for secretion management are also associated with risks, such as the development of pneumothorax through the use of a mechanical In-Exsufflator (MI-E) [435]. This pertains to both NMD and (especially) COPD patients, so that the technique is contraindicated in COPD patients with emphysema, also according to the manufacturer's instructions. In general, the physician is responsible for weighing up the risks against the advantages for each given measure.

\subsection{What Sort of Secretion Management Techniques}

Should Be Applied in Invasively Ventilated Chronic

Obstructive Pulmonary Disease Patients?

The number of invasively ventilated patients with COPD is on the rise, with most of these having experi- enced weaning failure (Weaning Category $3 c$ [17]). Effective secretion management is of fundamental importance when the ciliary apparatus becomes impaired by chronic inflammation of the airways, augmentation of secretions, and the existence of an artificial airway. High-osmolarity saline $(\mathrm{NaCl} 3-6 \%)$ inhalation should be used for secretolysis, although bronchoconstrictory side effects can arise. For this reason, topical therapy with short-acting beta-sympathomimetics (through inhalation) should take place as a prophylactic measure before each saline inhalation session. The duration of inhalation is around 15-30 min, depending on the nebulizer device. Forced expiration through the cannula (so-called huffing) can then be carried out under the support of physiotherapy. Alternatively, the secretion can be removed by endotracheal suctioning, provided it is located in the central airways. To this end, reference can be made to the recommendations by the American Association of Respiratory Care (AARC), which, based on evidence from paediatric studies, suggest strict endotracheal suctioning and the avoidance of deep endobronchial suctioning in adults [115]. This is because only the right bronchial system is usually probed, and despite the use of non-traumatic suction catheters, there is a clinically relevant risk of deep airway mucosal membrane damage with bleeding and relatively more secretion build-up. 
For extremely tough secretions or intolerance to saline inhalations, oscillation therapy for secretolysis and secretion transport in the central airways can be applied. Highfrequency oscillations can reduce the viscosity of bronchial secretions [436] and strengthen expiratory flow more than inspiratory flow [437], whereby the resonance effect can be even further reinforced by selecting an oscillation frequency of 10-15 Hz. Fornasa et al. [438] were able to show that low oscillation frequencies are actually responsible for the expansion of intrathoracic volume, while higher oscillation frequencies promote secretion mobilisation. The application of oscillations can take place either endobronchially or transthoracically. The prescription of appropriate aids and resources is currently possible on a case-by-case basis only, based on statements for individual reasons as well as the ineffectiveness of other cheaper therapeutic measures. Clear evidence for the superiority of oscillatory devices in comparison to other physiotherapeutic measures is currently unavailable, due to a lack of plausible, adequately powered studies [439].

\subsection{How Should Secretion Management in}

\section{Neuromuscular Disease Patients Be Carried Out?}

Accumulation of secretions in the airways can be detected early via a drop in oxygen saturation, which is measured at selective intervals by pulse oximetry. The oximeter feedback method developed by J. Bach is based on the fact that young patients with NMD do not have clinically relevant lung disorders and hence normally have an $\mathrm{SpO}_{2}$ $>95 \%$ [420]. The application of one or more methods for secretion elimination is recommended when $\mathrm{SpO}_{2}$ falls below $95 \%$ or by $2-3 \%$ in relation to the individual patient's best reading in pre-existing lung diseases. This explains the necessity for supplying the patient with a pulse oximeter for selective $\mathrm{SpO}_{2}$ measurements, in addition to resources for improving cough capacity.

Secretion management in NMD (Fig. 8) comprises measures for increasing intrapulmonary volume (e.g., air stacking [440], glossopharyngeal breathing, or manual hyperinflation) and measures for increasing expiratory flow during coughing (e.g., assisted coughing techniques or the application of mechanical coughing aids) [441445] (Table 11). Alternatively, an intermittent positive pressure breathing (IPPB) device can be used successfully, especially in children [446]. Similarly, high-frequency vibrations in combination with improved humidification of the airways can support both secretion mobilisation as well as centrally directed secretion transport. Even hyperinflation with an inspiratory pressure of 30-40 mbar with
Table 11. Secretion management in neuromuscular diseases help with secretion removal

Manual hyperinflation of the lungs (air stacking)

Increase in intrathoracic volume by breathing through a ventilation bag or volume-controlled ventilation device Glossopharyngeal breathing

IPPB through specialised ventilation machine functions

Manually assisted cough

Active pressure into the epigastrium or bilaterally at the costal arch for surge-like cranial shifting of the diaphragm at the advent of coughing

Mechanically assisted cough (MI-E)

Active inflation of the lung through excess (positive) pressure, followed by the application of sufficiently high negative pressure for secretion removal.

a special ventilation device is effective in improving PCF [447]. The establishment of an indication as well as the specification of parameters (particularly pressure and time settings) are primarily carried out by a physician. The initiation of therapy and briefing on the selected techniques can be performed by experienced personnel (doctor, respiratory therapist, nurse, or physiotherapist); proper training of the patient and ambulatory care personnel/relatives is paramount.

The attainable PCF is most often highest with the use of an MI-E [376, 404, 448, 449], followed by assisted coughing and non-supported coughing at the level of maximal insufflation capacity [450-452]. The MI-E can be applied either non-invasively via a mouthpiece or fullface anaesthesia mask (with valve), or invasively via an endotracheal tube or a tracheal cannula. It should be noted for invasive ventilation interfaces that as the tracheal cannula diameter decreases, so does the target maximal peak expiratory flow value; therefore, the applied inspiratory and expiratory pressures should be increased [453]. The performance of the various mechanical coughing aids can differ considerably with nominally equivalent pressure settings [454, 455]. For this reason, swapping the device through the provider or health insurance company for another from a different manufacturer is not possible without renewed testing of its efficiency and (if applicable) adaptation of the settings.

The use of mechanical coughing aids in children has demonstrated that minimal insufflation periods of $>1 \mathrm{~s}$ are required for adequate filling of the lungs [456]. Despite the somewhat suboptimal adherence in outpatient use, these devices should be considered as a supplement 
Fig. 8. Algorithm for secretion management in neuromuscular diseases.

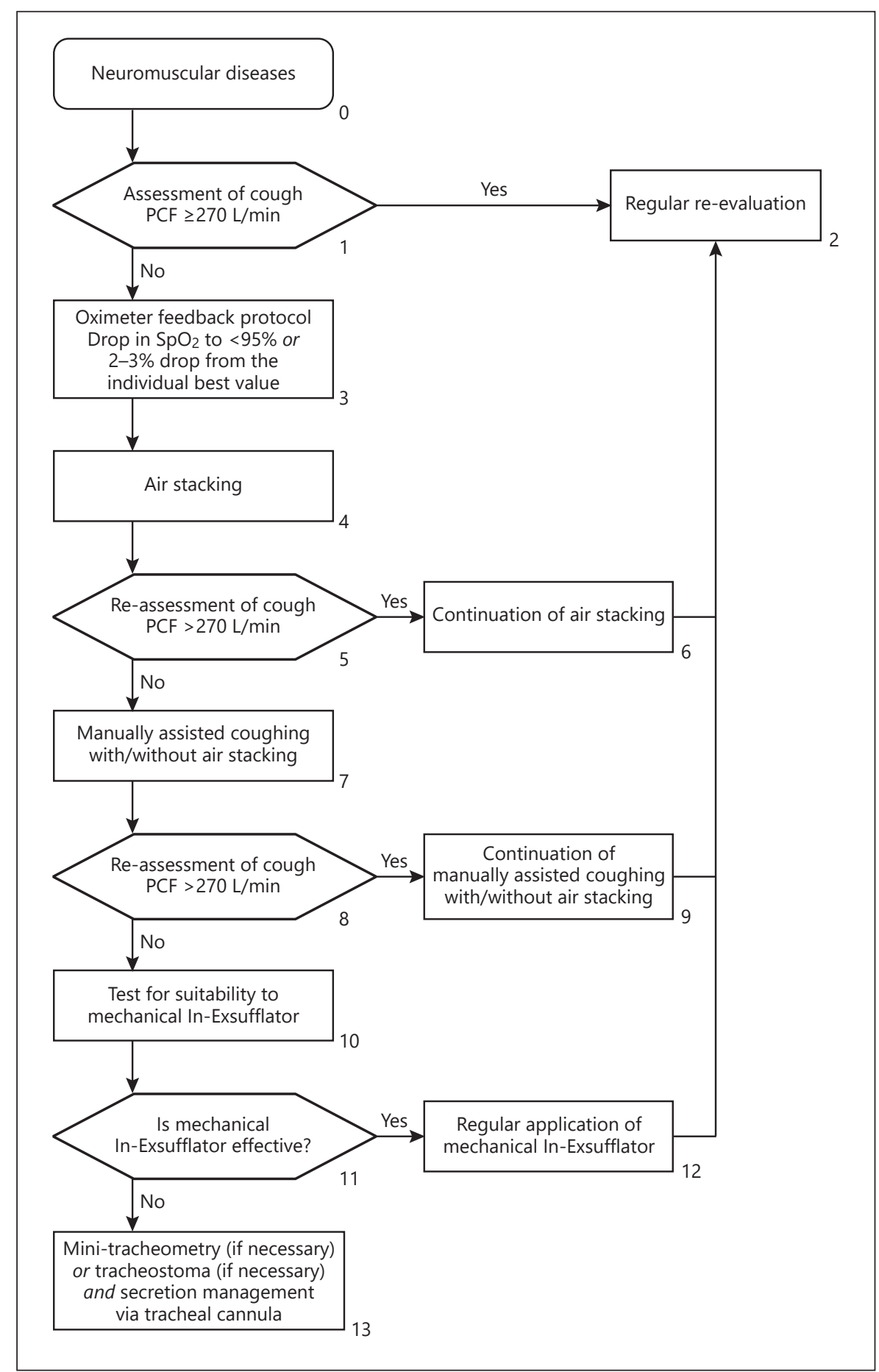

to HMV therapy in patients with reduced PCF, and thus prescribed accordingly [457]. Therapy adherence can be potentially enhanced through competent coaching of the patient and care team, effective therapy set-up, and the implementation of user-friendly devices such as those with synchronisation assistance via trigger options. Exact details about the optimal frequency of MI-E use are not available due to a lack of studies in this area. The number of applications should be based on the secretion load of the patient, thus allowing the airways to be cleared. The 
authors recommend a minimum therapeutic application of 3-4 times daily and additional use to be carried out as required (see oximeter feedback method). One application consists of a maximum of $4-5$ cycles. For practical use in adolescents and adults, inspiratory and expiratory pressures of at least 40 mbar at the device outlet are recommended [458], as is the use of (i) a bacteria filter, only if a catheter mount has been renounced upon for noninvasive application, and (ii) a straight catheter mount for invasive application [455]. A catheter mount tube with an opening for suction should only be envisaged in the event of extreme secretion loading, in order to suction out the secretion from the tracheal cannula during MI-E application. Set-up of inspiratory and expiratory times is based partly on subjective tolerability and partly on the remaining coughing capacity: if some coughing is still possible, the expiratory time can be shorter than the inspiratory time (as with normal coughing); if a cough is lacking, the expiratory time should be at least as long as the inspiratory time, since expert experience has demonstrated that liquid secretions can primarily become shifted by inspiratory pressure into the lung periphery, with centrally directed mobilisation only first appearing at the conclusion of the (non-physiological) longer expiration period. Consistent therapeutic application aims towards multiple applications with short breaks in between. The use of an individually adapted secretion management program can, in isolated cases, not only delay the time point of mechanical ventilation [440] and allow successful initial undertaking of NIV but also avoid hospitalisation $[96,388]$ and extend survival [442].

It is important to critically evaluate the efficacy of the selected type of secretion management program, educate patients and nursing/care support personnel in detail especially about specific and advanced techniques for dealing with infections of the lower airways - and integrate the general living circumstances; e.g., mobile patients who require an MI-E device need to be provided with an external battery. When hospitalisation is required, it is advisable to seek out a specialised clinic with knowledge of the different forms of non-invasive secretion management, in order to avoid intubation.

Further tools such as thoracic percussion or oscillation are also commonly used for secretion management; however, valid data on the effectiveness of these measures in NMD patients are not available. However, when contraindications for mechanical coughing aids exist, e.g., when pneumothorax is only conservatively treated, the abovementioned techniques can be prescribed if there is evidence for effective secretion management. In individual cases, failure of non-invasive secretion management can be satisfactorily controlled through the installation of a mini-tracheotomy with subsequent regular tracheal suctioning, without the need for a definitive tracheostoma.

Recommendations

- In neuromuscular disease patients with impaired cough and invasive ventilation, secretion management is obligatory and must be carried out regularly and adapted to the underlying disease.

- The indication for a mechanical In-Exsufflator device must be established by a physician with ventilation competency, who also then takes over the responsibility for the patient's therapy. Adaptation of the mechanical In-Exsufflator to the patient can be performed by a respiratory therapist.

- Switching between different types of mechanical In-Exsufflators that are not structurally identical may not be carried out without renewed testing of the effectiveness and patient compatibility of the devices; this is because the performance of each device may differ significantly.

- Secretion management in chronic obstructive pulmonary disease patients with high secretion load must include measures for secretolysis (medicinal/non-medicinal) and measures to ease coughing such as special coughing techniques (huffing) and positive expiratory pressure systems.

- Mechanical In-Exsufflators should not be used in chronic obstructive pulmonary disease patients.

- Suctioning in invasively ventilated patients must take place strictly via endotracheal placement of the suctioning catheter (max. $1 \mathrm{~cm}$ beyond the cannula), independent of the underlying condition.

\section{Spinal Cord Transection}

\subsection{Which Criteria Are Useful for Deciding between} Invasive and Non-Invasive Ventilation?

In principle, spinal cord transection patients who are dependent on mechanical ventilation can be either invasively or non-invasively ventilated. Since the decision for how this is ultimately done depends on a number of factors, no definitive instructions can be provided. Instead, the approach to HMV therapy should be based on what is best for each individual patient.

However, the decision can be guided by the particular clinical circumstances (e.g., acute or chronic spinal cord injury status, duration of ventilation, accompanying diseases/injuries, patient compliance).

\subsection{When Is Invasive Mechanical Ventilation \\ Necessary?}

Given that high-level spinal cord transection patients can experience protracted respiratory failure as well as the typical problems associated with long-term ventilation 
via oro- or nasopharyngeal tubes, a dilatative or surgically applied tracheotomy is often necessary to provide invasive ventilation access. The advantages of this are that analgosedation can then be withdrawn, allowing the conscious patient to be integrated sooner into the first phase of quadriplegia-specific therapy (wheelchair mobilisation; speaking and feeding during ventilation; weaning, if applicable).

Tracheotomy is generally recommended for those with cervical spinal cord transection when [459]:

- There is complete cervical motor paralysis corresponding to Type A and B on the ASIA Impairment Scale (AIS) [460] (Table 12)

- VC is $\leq 500 \mathrm{~mL}$

- The Injury Severity Score (ISS) is $>32$

- The $\mathrm{PaO}_{2} / \mathrm{FIO}_{2}$ ratio is still $<300,3$ days after the advent of ventilation

Studies have further shown that a so-called early tracheotomy $(<10$ days after the onset of paralysis) can shorten both the time spent in the intensive care unit as well as the entire ventilation period (provided that weaning is successful). This, however, influences neither the rate of pneumonia nor the overall duration of treatment [461].

In patients with persistent chronic spinal cord injuries requiring $>12 \mathrm{~h} /$ day of ventilation, the results of longterm studies show that $>90 \%$ of these patients opt for a tracheotomy and hence the continuation of invasive ventilation (even when NIV would generally be possible) [462]. The reasons for this are (i) easier secretion management in patients with reduced or absent coughing, (ii) unrestricted motor function of the facial muscles and hence the associated speech-related function, and (iii) poor tolerance of the ventilation mask for various reasons.

\subsection{What Should Be Noted for Non-Invasive}

Ventilation of Patients with Spinal Cord Transection?

The advantages of NIV are generally deemed to be the potential avoidance of intubation and tracheotomy, with the absence of surgically related complications and the preservation of the body's own ability to filter and warm tidal air. Furthermore, the potential long-term complications of tracheotomies and inserted tracheal cannulae can be avoided [463]. Coughing support in the context of secretion mobilisation generally includes the prescription of MI-E.

The prerequisites for NIV in patients with high-level spinal cord transection injury are [463]:

Treating Chronic Respiratory Failure with Invasive and Non-Invasive Ventilation
Table 12. The American Spinal Injury Association (ASIA) Impairment Scale (AIS)

\begin{tabular}{ll}
\hline $\begin{array}{l}\text { ASIA } \\
\text { Impairment } \\
\text { Scale (AIS) }\end{array}$ & Neurological deficit \\
\hline A & $\begin{array}{l}\text { Complete loss of motor and sensory function at } \\
\text { S4/S5 }\end{array}$ \\
\hline B & $\begin{array}{l}\text { Complete loss of motor, incomplete loss of } \\
\text { sensory function at S4/S5 }\end{array}$ \\
\hline C & $\begin{array}{l}\text { Incomplete loss of motor function at S4/S5, } \\
\text { average muscle grade below the neurological level } \\
\text { is }<3\end{array}$ \\
\hline D & $\begin{array}{l}\text { Incomplete loss of motor function at S4/S5, } \\
\text { average muscle grade below the neurological level } \\
\text { is } \geq 3\end{array}$ \\
\hline E & No motor or sensory deficits \\
\hline
\end{tabular}

- Functional preservation of facial and pharyngeal muscles

- Patient cooperation

- Unobstructed patency of the upper airways Contraindications are [464]:

- A lack of patient cooperation

- Increased risk of aspiration when protective reflexes are lacking

- Obstructions of the upper airways

- Secretion retention that cannot be controlled under non-invasive conditions

- Pressure ulcers associated with the ventilation mask Aerophagia and the resulting increase in intra-abdominal pressure can occur as an unwanted side effect [465]. The extent of the impaired gut motility that is commonly present in patients with spinal cord transection injuries can be increased (paralytic ileus) and/or lead to ruptures of the stomach or intestine. Patients lacking arm, hand, or finger function require adequate technical surveillance in addition to permanent clinical monitoring. The recommendations outlined in Chapter 8.6 also pertain to the transition of these patients into the home setting.

\subsection{What Distinguishes Spinal Cord Transection}

Paralysis from Other Types of Paralysis?

Spinal cord transection is characterised by damage to the spinal cord tissue at different levels, with varying degrees of motor, sensory, and autonomic impairment. Additional direct consequences are impaired urinary 
bladder and intestinal functions, spinal spasticity, deafferentation pain/dysesthesia and perturbed vegetative regulation [466-469]. Depending on the functional level and extent of the transection, different forms of respiratory failure with partial or complete requirement for ventilation may arise [470-472].

Accompanying diseases and/or injuries can represent further causes of ventilation dependency. Nevertheless, the special characteristics of spinal cord transection patients also apply to this group of patients, irrespective of the cause of ventilation dependency, since the consequences of spinal cord transection are generally so extensive that completely different standards need to be taken into account, even in patients undergoing mechanical ventilation for other reasons $[473,474]$.

\subsection{Which Therapy Objectives Have the Highest}

Priority in Patients with Spinal Cord Transection?

Patients with spinal cord transection paralysis are high-priority and contingent upon lifelong medical care that stems from the following general therapy goals:

- To ensure survival.

- To improve somatic status, or, at the least, to prevent overall deterioration (including that of possible complications), and to treat accordingly.

- To improve or at least to prevent the deterioration of mental status, since the indispensible condition for somatic improvement is stabilisation (at the least).

- To recognise and avoid the complications associated with spinal cord transection paralysis (e.g., pneumonia, decubitis, spasticity, autonomic dysreflexia). For example, high-grade spasticity can hinder ventilation to the extent that pharmacological spasticity attenuation (intrathecally, if applicable) may be necessary. The specific therapy goals are:

- Daily wheelchair mobilisation

- Restoration/preservation of speaking ability (even under mechanical ventilation)

- Assurance of communication ability

- Restoration of the greatest possible level of independence in daily life activities and in occupational and social integration

\subsection{What Needs to Be Observed for Nursing Care in} the Home Setting?

Permanent clinical monitoring by qualified nursing staff and the securing of all selectively required, diseasespecific care measures is ensured through a nursing care service that meets the legally required quality standards for structure, processing, and outcome $[475,476]$. This also applies to the case of NIV therapy in patients with loss of arm, hand, or finger function. Since the line between basic and therapeutic care can be blurred in patients with spinal cord paralysis, high medical expectations should be placed on the professional qualifications of the outpatient service.

The possibility of incorporating a patient's relatives into the care program should be considered on a case-tocase basis, since they can often be emotionally affected (especially in emergency situations) and hence not always be relied upon to securely carry out the necessary therapy measures. It should also be ensured that a second person is available for carrying out basic care duties (extensive body care, transfers, etc.) when the patient has a ventilation dependency and mobility-limiting factors exist (e.g., obesity, contractures, spasticity, dysregulation) [477].

\subsection{What Sort of Technical Aids Are Required for}

Disease Monitoring in Patients with Spinal Cord

\section{Transection?}

Due to the above-described shortcomings, patients with spinal cord transection paralysis are constantly dependent on external support for all aspects of daily life. Therefore, permanent technical monitoring is required for maintaining vital functions in addition to continuous clinical surveillance.

\subsubsection{Pulse Oximetry und Capnometry}

Continuous pulse oximetry is required for both partially and completely ventilated spinal cord transection patients, in cases where the patient cannot detect, report, or independently eliminate a disruption to the ventilation process. Lapses can be detected early and potentially corrected through regular capnometric measurements, before the patient becomes hypoxemic or exhausted from stipulated periods of spontaneous breathing. The set-up of ventilation devices without volumetry (e.g., diaphragm stimulation) can be adapted to the available $\mathrm{CO}_{2}$ measurements. In addition, the appearance of impaired gas exchange can be detected early at the onset of complications in the home setting (e.g., pneumonia).

Capnometric monitoring of spinal cord transection patients should therefore be supplied in the following cases:

- Patients undergoing invasive HMV via a single circuit system, in accordance with DIN EN ISO 80601-2-72: 2015 [45].

- Patients with an unstable ventilation situation due to clinically relevant vegetative dysregulation.

- Patients with additional central respiratory dysfunction. 
- When an implanted phrenic or diaphragm stimulator is applied (due to the absence of volumetry).

- For patients in whom a general change in ventilation parameters and/or modes are intended in the home setting.

- When spontaneous breathing performance is unpredictable and depends on the day-to-day form of the patient.

- For permanently or partly ventilated children, in consultation with the treating paediatrician.

\subsubsection{Spirometry}

Breathing volume during spontaneous and stimulating activities can be measured by a spirometer. This measurement can take place either via a mouthpiece or a tracheal cannula adapter. A prescription for spirometry is indicated when ventilation takes place through phrenic nerve/diaphragm stimulation (due to the absence of volumetry) or for monitoring spontaneous breathing during intermittent ventilation (assessment of breathing performance).

\subsubsection{Second Ventilation Device and External}

Battery

The indication for a second ventilation device and an external battery is always made independently of the daily ventilation times, since unforeseeable complete dependency on a respirator can occur due to the specific complications and characteristics associated with spinal cord transections. Furthermore, daily mobilisation of the patient into the wheelchair is the goal of therapy, hence the need for an external battery.

\subsection{When and for Whom Is Each Type of Diaphragm} Stimulation Method Appropriate?

In spinal cord transection lesions from $\mathrm{C} 0$ to $\mathrm{C} 3$, and in congenital or acquired central respiratory disorders, the subsequent systems can be implanted. Currently, there are 2 different systems for diaphragm stimulation.

\subsubsection{Phrenic Nerve Stimulation - Indirect}

Stimulation

The phrenic nerve stimulator has been a means of negative-pressure ventilation since the mid-1960s for patients with respiratory failure [477]. This entails the mediastinal implantation of electrodes on both phrenic nerves at the level of the 3rd-4th intercostal space, along with the entire set of receivers and cables. Stimulation takes place through transcutaneous induction under closed skin conditions.

Treating Chronic Respiratory Failure with Invasive and Non-Invasive Ventilation
Exclusion criteria for implantation are:

- Extensive cerebral defects with severe brain function disorders

- Significant pre-injury of the heart and/or lung parenchyma

- An unfavourable prognosis in terminally ill patients The prerequisites for implantation are intact alphamotoneurons for both phrenic nerves, undamaged peripheral nerves, and a functionally viable diaphragm muscle. Implantation is thus not possible with peripheral lesioning of the phrenic nerves, since transmission of the impulse cannot take place (e.g., previous thoracic trauma, infections, idiopathic phrenic nerve paresis).

International studies have reported a statistically significant drop in the rate of pulmonary complications, lower mortality rates, and an improvement in speech ability and quality of life [478-480]. The threshold and stimulation current values do not change over a longterm course, thus rendering the above-described systems suitable for long-term ventilation [481]. By saving on consumable materials, the extra expenses of the systems are paid off over the years [479]. Based on the unique form of stimulation, ventilation times are internationally quoted as $24 \mathrm{~h}$ /day in over $60 \%$ of cases [ $479,480,482]$.

\subsubsection{Diaphragm Pacing - Direct Stimulation}

In this procedure, the electrodes are directly introduced into the diaphragm muscle via laparoscopy, and the cable is led out of the abdomen [483]. Intact alphamotoneurons of both phrenic nerves, undamaged phrenic nerves, and a functionally viable diaphragm muscle are once again the prerequisites for implantation. Long-term stimulation of peripherally lesioned nerves is also not possible with this technique. This system is mostly applicable to the areas of temporary ventilation or respiratory support [484].

\subsubsection{Further Important Information about}

Diaphragm Stimulation

The particular operative complications associated with diaphragm stimulation are mentioned in the literature as possible phrenic nerve damage in phrenic nerve stimulation implantation $(<1 \%)$ and pneumothorax from the operation for diaphragm pacing $(<1 \%)[478,479,483$, 484]. In children and adolescents in whom the thorax is not yet fully grown, continuous 24 -h stimulation is not indicated until bony fusion of the thorax is complete (risk of attenuating tidal volume through chronic, mechanicalassociated thoracic retraction). In this case, patients generally undergo daily ( $12 \mathrm{~h}$ ) diaphragm (nerve)-stimulated 
ventilation, whereas invasive ventilation is carried out during the night as a means of thoracic protraction [479, $480,484]$. Both systems can replace external ventilation in patients with complete respiratory insufficiency, and hence fulfil the criteria for in- as well as outpatient/invasive HMV.

\section{Recommendations}

- Permanent pulse oximetry monitoring is necessary in partly and fully dependent mechanically ventilated patients with spinal cord transection.

- Capnographic monitoring of patients with spinal cord transection injuries is indicated in many situations in the home mechanical ventilation setting.

\section{Special Features of Paediatric Ventilation}

\subsection{What Are the Fundamental Principles of}

Long-Term Ventilation in Children?

The number of children and adolescents who require long-term mechanical ventilation has continuously risen in the last couple of years. The underlying diseases that lead to chronic respiratory pump insufficiency in childhood are generally complex and often connected with multiple disabilities, therefore requiring treatment in a specialised interdisciplinary centre. A therapeutic care program should anticipate the course of the underlying disease with all its potential complications and include infection prophylaxis, mechanical ventilation assessment, therapy for coughing insufficiency, sufficient nutrition support, and adequate management of complications and emergencies. To this end, particular focus should not only lie with the medical aspects, but also with the preservation of an acceptable quality of life and the greatest possible social participation of the affected children and their families [485-490].

\subsection{How Does the Diagnostic Approach in Children}

Differ to That in Adults?

In small children, but also older children with mental retardation, compliance-related examinations such as lung function testing and cough assessment cause difficulties or are even impossible to perform. Ventilation and gas exchange must then be directly measured. However, the problem of insufficient assessment of the respiratory reserve often remains. Since sleep-related respiratory disorders always precede manifest respiratory insufficiency, assessment of ventilation during sleep is desirable. If hypoventilation is detected during sleep, then diurnal decompensation is also possible; however, if respiration is compensated during sleep, a certain degree of respiratory reserve can be assumed. Fast decompensation of respiratory function requiring ventilation is possible during stressful events such as fever, airway infections, or operations, even in patients with inconspicuous somnological results.

The procedure for diagnosing chronic respiratory pump insufficiency is described in Chapter 5 and does not differ between compliant children and adults. In the presence of sleep-related respiratory disorders, an obstruction of the upper airways (e.g., through adenoids and/or tonsil hyperplasia) should be ruled out and treated if necessary. Here, it should be noted that children with inspiratory muscle weakness often do not snore. Neuromuscular diseases are often but not always accompanied by OSAS.

\subsection{What Are the Special Features Associated with Establishing an Indication for Non-Invasive Ventilation in Children?}

Paediatric diseases that are accompanied by hypoventilation/respiratory pump insufficiency and require ventilation are listed in Table 13. Indication for NIV is not based on the presence of a particular disease, but is rather dependent on the type and extent of respiratory impairment (OSAS, central respiratory regulation disorder, chronic alveolar hypoventilation, chronic or acute respiratory failure) [491].

The commonly alleged lack of cooperation in children is generally not a problem within an experienced centre. When the indication is correct and ventilation therapy is customised to the special needs of the child, the majority of children benefit from the therapy and tolerate, or even desire, mechanical ventilation therapy. This holds particularly true when children experience a perceivable improvement in their complaints through mechanical ventilation therapy.

\subsection{How Is the Indication for Tracheotomy and Invasive Mechanical Ventilation Established in Children?}

Common indications for the insertion of a tracheostoma in infants and small children are [492]:

- Obstruction of the upper airways through conditions including congenital malformation syndromes, vocal cord paresis, subglottic stenosis, tracheomalacia, traumas, progressive neurological diseases with bulbar paralysis, cerebral palsy.

- Diaphragm paralysis after heart surgery for a congenital heart defect. 
- Congenital and acquired pulmonary diseases such as pulmonary hypoplasia and bronchopulmonary dysplasia.

- Congenital and acquired NMD.

The distinctive feature in childhood is, however, that the tracheotomy represents a serious obstacle for ongoing child development. The insertion of a tracheostoma in an infant or small child particularly impairs speech development and swallowing, making intensive supervision and support necessary. Daily activities such as swimming are only possible on a very limited basis and must ultimately be ensured through constant accompaniment (kindergarten, school) by a qualified nurse.

Since a tracheostoma also represents a significant intrusion to the child's body image and a substantial burden for the patient's relatives, the indication for tracheotomy in childhood should essentially be made on a restricted basis. As is also the case for adults, all options for NIV should be exhausted before the insertion of a tracheostoma in children.

In some cases, however, the insertion of a tracheostoma can be necessary for anatomical reasons or because NIV is not possible. A ventilation duration of $>16 \mathrm{~h} /$ day also often leads to the necessity for a tracheotomy. In particular situations such as craniofacial dysmorphia, tracheostenoses/tracheomalacia, and in children with bronchopulmonary dysplasia, there is a very good chance that the tracheostoma can be closed again in the later stages of childhood [493].

The process of deciding for or against a tracheostoma is always shaped by individual attitudes and the ethical or even religious convictions of the child, its parents, and the team of therapists. To this end, it is common for conflicts to arise, especially if the affected child suffers from a progressive underlying disease or if there is an unfavourable prognosis for development. The treating physicians often face the dilemma of not alleviating but rather prolonging the suffering of their patients. For parents, however, deciding against a tracheostoma is especially difficult when this means that the end of the child's life is imminent without tracheotomy. The enlistment of clinical ethical committees and palliative care teams is often very helpful in this formidable and burdening decision process [493497].

15.5 What Kind of Evidence Is Available for the Effects of Long-Term Mechanical Ventilation in Children?

Even though data are largely restricted to non-randomised studies, it appears certain that children with congenital central hypoventilation syndrome (CCHS)/
Table 13. Paediatric diseases prone to chronic hypercapnic respiratory failure

Central respiratory regulation disorders

- CCHS or Ondine's curse

- Acquired central hypoventilation through brain stem affection (e.g., after tumour, trauma, bleeding, or encephalitis)

- Degenerative diseases or CNS tumours

- Stenoses of the cranio-cervical transition (among others Arnold-Chiari malformation), syringomyelia

Restrictive ventilatory disorders

- NMD (spinal muscular atrophy type I-III, SMARD, muscular dystrophies including Duchenne, structural myopathies, central core myopathy, nemaline myopathy, myotonic dystrophy, other myopathies)

- Mitochondriopathies

- High-level spinal cord transections (including post-trauma, bleeding, tumour, myocele, transverse myelitis)

- Kyphoscolioses (idiopathic or secondary to NMD, infantile cerebral palsy, myelomeningocele)

- Microthorax (asphyxiating thoracic dysplasia/Jeune syndrome, osteogenesis imperfecta, achondroplasia)

- McCune-Albright syndrome

- Lung parenchymal diseases (bronchopulmonary dysplasia, pulmonary hypoplasia, cystic fibrosis, post-ARDS status)

Lung parenchymal diseases

- Bronchopulmonary dysplasia

- Pulmonary hypoplasia

- Cystic fibrosis

- Non-cystic fibrosis bronchiectasis

- Defective status post-ARDS and post-pneumonia

Obstructive sleep apnoea and obstructive hypoventilation

- Alimentary morbid obesity

- Prader-Willi-Labhard syndrome

- Tonsillary hyperplasia

- Soft palate dysfunction

- Laryngomalacia

- Trisomy 21

- Macroglossia

- Storage diseases such as mucopolysaccharidoses

- Apert syndrome

- Pierre-Robin sequence

Paediatric diseases that are associated with hypoventilation/respiratory pump weakness and may require ventilation. CNS, central nervous system; SMARD, spinal muscular atrophy with respiratory distress; ARDS, acute respiratory distress syndrome.

Ondine's curse or progressive NMD benefit from HMV [498]. HMV normalises the respiratory deficit and optimises blood gases, improves sleep, reduces morbidity and hence the number of hospital treatment days, reduces mortality, and enhances the quality of life. Even though systematic studies of other diseases are lacking, a similar 
outcome can at least be assumed for diseases in which the chronic respiratory failure does not have an underlying lung parenchymal component [282, 375, 452, 499-502].

\subsection{What Should Be Particularly Noted about Home} Mechanical Ventilation in Children?

HMV in children is associated with a multitude of special characteristics as well as difficulties. Noteworthy points include the absent or restricted ability to communicate and cooperate in younger and mentally retarded children, as well as problems with the technical equipment, which is often not designed for mechanical ventilation in small children.

The following points should be taken into account when choosing appropriate ventilation machines and masks for NIV in children [503-505]:

- Children with muscle weakness may not be able to sufficiently trigger particular types of ventilators. Ventilation volumes are very low, especially in younger children; at the same time, children exhibit irregular breathing frequency and depth.

- The need for ventilatory support changes constantly; it is higher during the awake state than during sleep. During sleep, the need is dependent on the stage of sleep. The need can escalate in the event of fever or infection.

- Not all ventilators are approved or appropriate for use in small children; many devices only generate reliable tidal volumes with a body weight of $30 \mathrm{~kg}$ onwards or cannot be triggered reliably during NIV.

The range of customised nasal masks for infants and small children has improved significantly. Masks that are not specifically designed for children often harbour a relatively high dead space and frequently have a poor fit, especially in younger children (small face, facial deformities in some diseases). Therefore, in special cases the production of a so-called individual mask is inevitable.

The risk of developing a midface deformity is increased with masks that have a high contact pressure. Small children, as well as those with muscle diseases and immobility, cannot remove the ventilation mask themselves in emergency situations (equipment errors, electricity failure, nausea/vomiting). Therefore, oronasal or full-face masks should be renounced upon in children, where possible. Preference should generally be given to nasal mask ventilation. In the event of pronounced oral leakage, however, sufficient ventilation is only possible in some cases with a full-face mask. To minimise potential harm, adequate surveillance of the child during ventilation is mandatory [506].
Special attention is required during the transition from paediatric to adult patient. To this end, seamless hand-over of the patient with all of the associated speciality disciplines should be ensured. Since most patients undergoing the transition into adulthood suffer from NMD, reference should be made to Chapter 12.5 .

15.6.1 What Are the Special Technical Requirements Associated with Home Mechanical Ventilation in Children?

The following requirements are derived from the special features outlined in Chapter 15.6:

- The ventilator should be able to reliably generate a low tidal volume.

- A sensitive trigger is necessary to adapt the assisted ventilation function to changing ventilatory needs, without a significant increase in the work of breathing.

- Devices with pressure presets can adapt to a variable breathing pattern and are therefore almost always more appropriate than devices with volume presets.

- A customised mask should be made when an appropriate commercial mask is not available. Readaptations of the masks are more often necessary in childhood than in adulthood, due to growth.

15.6.2 What Needs to Be Considered for the Care of Tracheotomised and Invasively Ventilated Children? Depending on their body weight, children have a relatively high basal metabolism and high minute volume. The additional airway resistance of the small tracheal cannulae can lead to respiratory decompensation during spontaneous breathing or to a reduction in - or a complete lack of - spontaneous breathing ability. The risk of secretion build-up is inversely proportional to the inner diameter of the cannula. The high airway resistance of these cannulae increases exponentially, even with minor contaminations; life-threatening complications may arise.

Due to the relatively high breathing frequency of children, fluid loss via the airways is high; sufficient passive and/or active humidification of tidal air must be ensured to prevent complications. Hereby, active humidification during the night and passive humidification via an HME filter during the day has been shown to be successful.

As a prerequisite for speech development in infants and small children, cannulae should always be selected on the basis that they ensure adequate leakage via the glottis and that children still have the ability to vocalise under ventilation. A relatively large inner cannula diameter for reducing airway resistance, and a not-too-large outer 
cannula diameter for leakage compensation, can represent a contradictory situation that is not always easy to solve. To guarantee better speech development, a speaking aid should be implemented as early as possible in children with long-term or intermittent spontaneous breathing ability.

Unblocked cannulae are normally used for children undergoing ventilation. Aeration of the larynx during expiration generally allows vocalisation, prevents the accumulation of saliva, facilitates the act of swallowing, and prevents aspiration [507]. The use of cuffed tracheal cannulae should be limited to children with pronounced swallowing disorders and a high risk of aspiration. However, even cuffed cannulae do not reliably safeguard against aspiration. The application of cuffed cannulae can sometimes be required in difficult ventilation situations (especially those with high leakage). For routine application, cuffed cannulae with an inner diameter of $4 \mathrm{~mm}$ have already become available. A cuffed tracheal cannula must not be used for extended periods without the cuff, because the unblocked cuff of most cannulae has sharp edges that can lead to tracheal injuries. Cannulae with a less bulky cuff (Tight-to-Shaft, TTS) that are blocked with distilled water and do not have firm edges in the unblocked state are now commercially available.

Cannula-associated emergencies occur more often than in adulthood. Infants and small children in particular can accidentally remove the cannula themselves. Small parts can be aspirated through the cannula or become lodged in the tracheal lumen [508].

15.6.3 What Are the Special Features Associated with the Care of Children in the Home Mechanical Ventilation Sector?

Specialist expertise should preferably be enlisted for treatment of the underlying disease (e.g., in a neuromuscular clinic) or in the event of complications (e.g., orthopaedics). Ideally, children are cared for by a multidisciplinary team both in the hospital and at home, and this can be supplemented by paediatricians, social workers, and family assistance, if required. In addition, for safe HMV and integration of the somewhat complicated therapy into the daily life of the affected families, a structured transition management program as well as support for the family during the hospital stay are essential. This holds especially true for invasive HMV and dependent non-invasively ventilated children. Further possibilities for support are available through self-help organisations. Contact with other affected families is also very helpful.

Treating Chronic Respiratory Failure with Invasive and Non-Invasive Ventilation
If a nursing care service is involved, staff should inform themselves about the individual features of the therapy and the needs of the patient and family whilst the child is still in the clinic. Provided the care of a ventilation-dependent child occurs through a nursing facility, the division management and care program should be ensured primarily through paediatric nurses (public health nurses as second priority), with the additional qualifications in paediatric ventilation care described in Chapter $6.4[220,221,495,508-512]$.

\subsection{What Should Be Observed during the Monitoring of Ventilated Children?}

HMV children should have access to a pulse oximeter. Continuous pulse oximetry during ventilation is obligatory for the detection of complications in tracheotomised children, and for helpless children who are ventilated via a full-face mask. At the same time, carers are often disturbed by frequent false alarms and this can interfere with their ability to concentrate on real complications.

False alarms and inaccurate measurements that result from movement artefacts or weak perfusion can be reduced through the use of an appropriate adhesive sensor and a pulse oximeter with an artefact-minimising function.

The ad hoc measurement of oxygen saturation is part of the emergency response plan. This provides parents with the possibility of objectively assessing the respiratory situation in cases of airway infection, fever, increased secretions, coughing, dyspnoea, and enforced breathing. Intervention is normally not required when $\mathrm{SpO}_{2}$ is $>95 \%$ during spontaneous breathing of ambient air. For $\mathrm{SpO}_{2}$ values between 90 und 95\%, the patient should initially intensify mask ventilation and/or assisted coughing. Once the situation stabilises, further outpatient treatment can be carried out. If oxygen saturation drops below $90 \%$ despite mechanical ventilation, the ventilation centre should be immediately informed or visited.

It has been demonstrated that the number of pneumonia cases and hospital stays can be reduced through a similar patient protocol [420]. Children without previous LTOT and in whom stabilisation of the respiratory situation cannot be achieved through the above-described procedure should not receive oxygen at home under these circumstances. The administration of oxygen in particular situations masks the progression of the underlying problem and delays diagnosis and appropriate hospitalbased treatment. Home $\mathrm{PCO}_{2}$ monitoring is justified on a case-to-case basis. 
15.8 What Type of Clinical Check-Ups Should Be

Carried Out in Home Mechanically Ventilated

Children?

Regular check-ups are necessary in order to ensure successful HMV in children. The ventilation therapy that has been initiated should be regularly adapted to the growth of the developing child and the progression of the disease. It is also worthwhile carrying out an in-hospital check-up examination within 1-3 months of starting ventilation therapy (nocturnal $\mathrm{CO}_{2}$ monitoring, synchronisation of ventilation), repeating these check-ups at 6-month intervals thereafter.

A paediatric cardiological examination that checks for right ventricular strain, pulmonary hypertension, and cardiac insufficiency (in the context of the underlying disease) should take place once a year. A tracheoscopy should be performed at least once a year in tracheotomised children to make valid sizing adjustments to the cannulae and enable the recognition of complications (tracheal granuloma, ulcers).

\subsection{What Are the Special Features of Secretion Management in Children?}

Children with NMDs particularly develop coughing insufficiency as a consequence of pulmonary and thoracic restriction, as well as the participation of the expiratory and abdominal muscles that are required for coughing. In children with spinal muscular atrophy, clinically relevant coughing insufficiency can already appear years before the manifestation of respiratory failure. This is because the trunk muscles required for expiration are more strongly affected than the inspiratory-related diaphragm muscle [494].

Screening of coughing function through measurement of PCF is therefore obligatory in children with the ability to cooperate. This can be easily measured by a commercial Asthma Peakflowmeter device (with the application of a nose peg). A PCF $<160 \mathrm{~L} / \mathrm{min}$ in children and adolescents is a strong predictor for the emergence of a type of pneumonia that needs to be treated in hospital; a PCF $>200 \mathrm{~L} / \mathrm{min}$ represents a negative predictor for recurrent chest infections [513].

The coughing impulse can also be effectively supported in children through mechanical assistance. Through IPPB-assisted coughing, the various MI-E and special mechanical inflation manoeuver functions of some ventilation devices were able to demonstrate improved coughing in children with good acceptance levels. The technique of mechanically assisted coughing should be well practiced with the child and supplemented by physiotherapy [446, 447, 514, 515].
Regardless of the indication for mechanical ventilation, children with frequent infections of the upper airways and a reduced coughing impulse should learn the technique of mechanically assisted coughing and be provided with the corresponding resources, if effectiveness is documented. Since there are no data on the effectiveness of vibration vests in children, the use of this resource should be restricted to exceptional cases.

\subsection{What Are the Special Features Associated with}

Home Mechanical Ventilation Therapy for Central

Hypoventilation Syndromes?

Hypoventilation syndromes (central hypoventilation syndrome, CHS) are generally understood as alveolar hypoventilation that predominantly occurs during sleep. Acquired CHS can arise as a consequence of conditions that include encephalitis, traumas, and central masses. The more common form is CCHS, which is also known as Ondine's curse. CCHS can be diagnosed on the basis of the disease-defining PHOX2B gene. Different mutations have been detected in the 3 introns of this gene [516, 517]. The type of genetic variant correlates rather strongly with the clinical manifestation of symptoms, and hence can also serve as a predictor of the patient's prognosis [518]. A diminished or completely abolished $\mathrm{CO}_{2}$-respiratory response is characteristic for CHS. The extent of dysregulation ranges from mild hypoventilation to apnoea with substantial hypoxemia lasting for several minutes. A short-term increase in alveolar ventilation typically arises during sleep at the point where $\mathrm{PaO}_{2}$ is $<30$ $\mathrm{mm} \mathrm{Hg}$ in the presence of hypercapnia.

CCHS can directly manifest postnatally; in this case, children should be placed on permanent ventilation in the first few weeks of life. Frequently, they cannot be weaned off mechanical ventilation, which is often the consequence of tracheotomy. Increasingly adequate breathing in the awake state can often develop later on so that HMV therapy can then be restricted to sleep. In some cases, the first symptoms of CCHS can even occur during the first few months of life or in adulthood [519]. If CCHS does not manifest directly after birth, hypoventilation frequently goes unrecognised and only becomes apparent through reticulocytosis, polycythemia, or pulmonary hypotension, with or without pulmonary heart disease [519].

In contrast to that in NMD, hypoventilation in CCHS is most pronounced in non-REM sleep. In daily clinical practice, this can lead to considerable fluctuations in $\mathrm{PCO}_{2}$ with alternating phases of hyper- versus hypoventilation. Negative-pressure ventilation and phrenic nerve 
stimulation are potential forms of mechanical ventilation for these patients, although they can be accompanied by an obstruction of the upper airways. Nowadays, NIV is even increasingly being prescribed to infants and small children. If this is not primarily possible, school-aged children can often be switched to NIV.

Oxygen therapy alone is contraindicated in this patient group. The type and mode of ventilation therapy should be adapted both to the situation and the patient's prognosis. Phrenic nerve stimulation is most often (but not exclusively) indicated for patients with a ventilation dependency during the growth phase [520]. Since most children are dependent on the ventilation therapy for survival, continuous monitoring of oxygen saturation (at the least) and $\mathrm{PtcCO}_{2}$ (if necessary) is essential.

\section{Recommendations}

- Due to their difference from adults, the care and ventilation of children with chronic respiratory failure must be carried out by a multidisciplinary team.

- Safe and successful home mechanical ventilation therapy must take into account the family and home environment of the patient and be accompanied by offers of medical, nursing, and psychosocial support.

- Continuous monitoring of oxygen saturation (at the least), and $\mathrm{PCO}_{2}$ (if necessary) must be carried out in children who are dependent on mechanical ventilation for survival.

- The development of paediatric competence centres that initiate home mechanical ventilation should be aimed at for better care of long-term-ventilated children.

\section{Ethical Considerations and Palliative Medicine}

\subsection{What Is the Significance of Ventilation at the End} of Life?

Palliative care is a measure for improving the quality of life in patients and their families who are confronted with the problems that accompany a life-threatening illness. This is achieved by prevention and relief of complaints via early detection measures, and careful evaluation and treatment of pain, as well as physical, psychosocial, and spiritual issues. Regarding the palliative medicine component (irrespective of the underlying diagnosis), reference can be made to the S3 Guideline for Palliative Medicine from the German Guideline Program in Oncology (AWMF Registration Number: 128/001OL) [521]. This document also contains important recommendations for care programs in palliative medicine, with a socalled care pathway for patients and relatives.

Patients who have chronic respiratory failure and are on HMV usually have a poor prognosis. For this reason, health-related quality of life carries particular significance. The physical quality of life under HMV therapy is most often rated by the patients themselves as reduced, whereas the mental and psychological quality of life is rated as good in some cases [522]. On one hand, HMV offers the chance to significantly improve both the extent of chronic respiratory failure and the quality of life [42] and can function as a palliative measure in the context of dyspnoea monitoring. On the other hand, it also comes with the risk of unnecessarily prolonging the patient's suffering and preventing him/her from dying with dignity at the end of a long illness [523].

Even the ventilation interface plays an important role at the end stages of life [524]. Since invasive or non-invasive ventilation interfaces can lead to a relevant impairment of quality of life, it should be assessed on a case-tocase basis whether a reasonable prospect exists for improving the quality of life through the use of an appropriate alternative ventilation interface.

\subsection{How Should End-Stage Patients Be Informed} about Their Options?

A patient-oriented clarification and information process is of central importance in advanced-stage diseases and/or rapidly progressing chronic respiratory failure. A dialogue should take place in which the physician informs the patient and his/her relatives about impending respiratory emergencies and therapeutic options for the final stage of the disease. The inclusion of the patient's caregivers in this communication process assumes the consent of the patient. If carers or health attorneys (see $\$ 1904 \mathrm{Abs}$. 2 BGB) are appointed, they should then be involved in this dialogue. A one-off discussion at the time of diagnosis is generally not adequate; ongoing regular informative and advisory conversations should also be made possible as the situation progresses, in order to create an atmosphere of trust and commitment. A practical approach on how to proceed can be devised, bearing in mind the actual/presumed wishes of the patient. Appraisals from the therapy team, the patient's caregivers, and (if available) the patient's representative should also be included in this process.

\subsection{How Should Communication between the Doctor} and Patient Take Place during the End-of-Life Phase?

From a medical perspective, the critical final stage of life is no longer about managing organ disease or technical challenges associated with medical devices, but rather about social competence and the ability to communicate. Regarding the communication between the physician 
Table 14. Subject domains relating to end-stage patients

Patient- and family-oriented decision-making

Emotional and practical support

Symptom/comfort-oriented care

Adequate pain and symptomatic therapy

Avoidance of unnecessarily prolonged dying

Autonomy of the patient

Reduction in the burden on relatives and support for the relationship between patients and relatives

Spiritual, emotional and administrative support from the care team

and patient, a paradigm shift has recently taken place, but has not yet fully been put into effect. The earlier prevailing paternalistic concept of the doctor-patient relationship, including the heteronomous control of the patient by the treating physician, is increasingly being replaced by a dialogue between the physician and the responsible, autonomous patient and his/her family, with priority given to the interests of the patient [525].

The modern doctor-patient relationship is based on a partnership, even in the final phase of life, where not only medical competence but also clear statements about the prognosis - especially in relation to end-of-life questions - and the duty of medical care remain unrenounceable. According to the guidelines for the care of terminally ill patients published by the German Medical Association (2011): "Informing the terminally-ill patient about his/her status and the possible course of action must be truthful, but should also be oriented towards the patient's situation and take into account any existing fears of the patient." The physician should also critically check that his/her own reservations about such a conversation do not influence the presumption of over-taxing the patient. The basic prerequisite for the mutual decision-making process is the exchange of information and transfer of knowledge between doctor and patient. Communication should correspond to the cognitive and intellectual abilities of the patient. However, successful communication is not only based on knowledge transfer, but should also address the patient's fears [526].

The decisive prerequisite for the successful care of terminally ill patients is close collaboration with the professional care personnel, which means integrating the nursing staff into the therapy team. In this context, so-called domains that relate to dealing with end-stage patients and their caregivers have been formulated in the literature (Table 14) [527, 528].
16.4 What Needs to Be Considered when Drafting an Advanced Health Care Directive?

It should be conveyed as early as possible to the patient, relatives, and caregivers that in the case of a respiratory emergency, the most secure way of preventing an undesired intubation with the potential for subsequent long-term ventilation is through a precise declaration of intent with concrete stipulations on how to act (advanced health care directive, AHCD). The AHCD should be formulated under expert (especially medical) advice. Finally, the AHCD should be documented. Patients and their relatives should be referred to the available information resources for the AHCD (e.g., brochures and events). The Internet site for the German Federal Ministry of Health (www.bmg.bund.de) is useful in this context [529].

Attention is drawn at this point to the principle of "advanced care planning" which is becoming increasingly established [530]. A once-drafted AHCD carries the risk of inadequately accounting for any alteration over time to the patient's originally documented declaration of intent. It is therefore recommended that the AHCD be updated like a log book in which discussions, wishes, and opinions are constantly documented.

\subsection{What Is the Procedure when Patients Are}

Incapable of Communicating/Expressing Their

Wishes?

When the patient is incapable of communicating or expressing his/her will, he/she is represented by a health attorney or a legally appointed guardian, who is normally a medically lay person. A health care proxy allows one person to authorize another to perform in an emergency situation some or all tasks on behalf of the appointer. The guardian or health attorney is required to express the actual or presumed wishes of a patient who is incapable of making decisions. In well-founded cases, and in consensus with the guardian of the incapacitated patient, gradual or abrupt withdrawal of treatment can take place even without the patient's permission, if this corresponds to his/her presumed wishes [531].

Next to the presumed wishes of the incapacitated patient (that are conveyed by the authorised person), the AHCD in particular sets the precedent for the actions of the persons involved. The rejection of a particular treatment (as expressed in an AHCD) is binding for the treating physician, as long as the situation at hand corresponds to the one that is described in the AHCD and there are no indications for a subsequent change of will. The AHCD becomes more binding for the treating doctor as the formulations of the patient's wishes become more detailed; 
this particularly holds true when a professional consultation is documented.

The AHCD should state, however, that under certain circumstances and with respect to the individual situation, the patient does not categorically reject a temporary intervention (e.g., tracheotomy for invasive ventilation) in the event of a potentially reversible crisis (e.g., in the form of treatable pneumonia).

\subsection{When Should Mechanical Ventilation Therapy \\ Be Minimised or Withdrawn Completely?}

If there is no hope even for the stabilisation of quality of life that corresponds to the expectation of a home-ventilated end-of-life patient, it is ethically justified to discuss and (if necessary) undertake a change in therapy goals in the form of therapy limitation or withdrawal $[179,532]$. However, the transfer of a patient to a hospice does not necessarily represent the indication for withdrawal of mechanical ventilation therapy; it can also be continued for the treatment of dyspnoea in a palliative context. To this end, coordination between the resources and facilities of the hospice and the treating palliative care physicians is useful.

"Withdrawing" therapy in this context means that ongoing therapy (e.g., mechanical ventilation and oxygen application) is gradually reduced and ultimately terminated. "Withholding" therapy means that a form of therapy indicated in other cases is not initiated.

The term "change of therapeutic goal" implies that the end-stage patient should receive all medical, nursing, religious, and spiritual treatment and care. This includes the following basic care measures:

- Appropriate placement of the patient

- Humane care

- Personal hygiene

- Relief of pain, dyspnoea, nausea, and other subjectively burdening symptoms

- Satisfying subjectively present hunger and thirst

Once mechanical ventilation has been started it should neither be forcefully or automatically continued nor must it be potentially "terminated" or "withdrawn." Instead, mechanical ventilation therapy should, like other treatments (medication, nutrition, infusion therapy, etc.), be checked regularly (e.g., daily), with strict consideration of the patient's will for its ongoing justification/indication.

In 1991, the German federal court decided that in the case of an unfavourable prognosis, and in accordance with the declared or presumed wishes of the patient, assisted dying through the non-initiation or withdrawal of life-extending measures (including ventilation) may be

Treating Chronic Respiratory Failure with Invasive and Non-Invasive Ventilation performed to allow a dignified course of dying (under effective pain medication, if necessary). In this context, there is generally a severe lack of information amongst many of those involved [533]. Doctors and nursing personnel should make their decisions based on knowledge of the current legal situation and are required to adequately inform themselves about the different terminology such as "killing on request."

Termination of mechanical ventilation in such a situation is designated as passive euthanasia in Germany. This is legally allowed and ethically justified, but should also be clearly distinguished from culpable "killing on request" [531]. In accordance with the guidelines of the $\mathrm{Na}$ tional Ethics Committee, the following notions are defined:

- Killing on request is defined in accordance with $\$ 216$ StGB as the situation in which somebody is assigned to the killing by the explicit and serious demands of the person to be killed and induces death in a directed and active manner. Killing on request is forbidden in Germany.

- The situation of letting somebody die is present when life-sustaining measures are refrained from, limited, or terminated/withdrawn - as long as this corresponds to the wishes of the patient or, in incapacitated patients, the originally expressed or presumed wishes. Letting somebody die is not a criminal act in Germany.

- End-of-life therapies carry the primary aim of relieving the patient's suffering. Through the use of strongly effective medication for the control of symptoms, a potentially unavoidable shortening of life can be legally tolerated (Bundesärztekammer, 2011). This type of end-of-life therapy is not a criminal act in Germany.

According to $\$ 217$ StGB (the intention of supporting another person's suicide or allowing, providing, or mediating in a business-like fashion the opportunity to commit suicide), the business-related assistance of suicide has to be distinguished from the above-described notions. People who do not act in a business-related setting and are either the relatives or close associates of the person mentioned in $\$ 217$ StGB section 1 are exempt from punishment.

\subsection{What Is the Nature of the Dying Process during or after Mechanical Ventilation?}

Disconnection from the ventilation machine is not invariably associated with immediate dying. Some patients exhibit low-level stabilisation following disconnection from the ventilator, and only die days or weeks later on a general ward, in a nursing facility, or ideally in a familiar 
Table 15. Principles of palliative medicine

Freedom from pain and agitation

No acceleration, but also no prolongation of the dying process Acknowledgment of life and death as physiological processes Integration of psychological and spiritual aspects Life support until the end, and support for relatives

Important principles of palliative medicine according to [536].

home setting [534]. However, since the majority of patients die in direct temporal connection with therapy withdrawal, there should be a special area/room available, in which the patients are enabled a dignified death in the presence of their relatives. The reality is far removed from this objective, however, and the responsible people must not refrain from requesting such facilities.

The dying process can be enabled not only through a definitive ending, but also through continuous reduction of the degree of mechanical ventilation support. In this respect, various concepts for the pharmacological management of mechanical ventilation therapy withdrawal exist, depending on the cultural surroundings. Dyspnoea, agitation, and pain should in any case be treated pre-emptively and in a symptom-oriented manner via application of potent analgo-sedatives [535]. It is reasonable not to abruptly end mechanical ventilation, but rather to gradually reduce the level of mechanical support to allow hypercapnia, acidosis, and progressive sedation and in turn avoid suffering during the dying process.

If the decision has been made to end mechanical ventilation in a patient, all available options as well as the principles of palliative care medicine as mentioned in Table 15 should be applied [536]. For the nursing component, reference can be made to the nursing guidelines for pain management, which optimise the interdisciplinary collaboration in pain therapy [537].

\subsection{What Are the Effects of Home Mechanical}

Ventilation in the Final Months of Life?

The last weeks and months of life at home under HMV therapy are frequently associated with difficulties, both for the patient as well as the care-giving relatives. An Italian multi-centre study surveyed the relatives of 168 deceased patients in the final 3 months of life under HMV [538]. The participation rate of $98.8 \%$ was very high, reflecting the relatives' need to discuss and share their experiences with the end-of-life situation of their relative. More than $80 \%$ of the patients experienced dyspnoea de- spite mechanical ventilation. Interestingly, this was markedly higher during NIV than invasive mechanical ventilation. In most cases, the patients seemed to be conscious both of their situation and prognosis. However, 54\% of the patients were hospitalised in the stage of final clinical deterioration and some even treated in an intensive care unit setting. Resuscitation measures were carried out in $27 \%$ of the patients. Furthermore, the study showed that many families experienced financial difficulties in relation to end-of-life care. It was therefore concluded that future studies on this subject are necessary and that options should be sought to improve quality of life in the final weeks of life, to avoid unnecessary hospitalisations and to consider the needs of the entire family [539].

For further consolidation of this topic, reference can be made to the detailed commentary on the ethical aspects of end of life in the S2K Guideline "Prolonged Weaning" [152].

\section{Recommendations}

- In very advanced or rapidly progressing chronic respiratory failure, patients and their relatives must be informed as early as possible about the potential imminent respiratory emergencies and the therapeutic options for the end stage of the disease.

- A partner-like relationship must take place between patient, physician, and nurse, even in the final phase of life, whereby medical competence but also clear statements about the prognosis, particularly in relation to questions about the end of life, and the duty of medical care remains indispensable.

- The rejection of a particular treatment as expressed in an advanced health care directive is binding for the treating physician, as long as the situation at hand corresponds to the one described in the advanced health care directive and there are no indications for a subsequent change of will. The content of the advanced health care directive should be regularly updated.

- As an alternative to the hitherto used terms "withholding" and "withdrawing" of ventilation, the term "change of therapeutic goal" should be used; hereby, the principles of palliative medicine should be applied using pre-emptive pharmacological therapy for dyspnoea, agitation, and pain, in combination with non-pharmacological therapy options.

- A private area should be available where patients are enabled a dignified dying process in the presence of their relatives.

\section{Acknowledgment}

We thank Dr. Sandra Dieni for her assistance in translating the manuscript.

\section{References}

For references, see online supplementary material (for all online suppl. material, seewww.karger.com/doi/10.1159/000488667). 
AHCD Advanced health care directive

ALS Amyotrophic lateral sclerosis

AWMF Arbeitsgemeinschaft der Wissenschaftlichen Medizinischen Fachgesellschaften e.V. (Association of the Scientific Medical Societies in Germany)

BGA Blood gas analysis

CCHS Congenital central hypoventilation syndrome/Ondine's curse

CHS Central hypoventilation syndrome

CIM Critical illness myopathy

CIP Critical illness polyneuropathy

$\mathrm{CO}_{2} \quad$ Carbon dioxide

COPD Chronic obstructive pulmonary disease

CPAP Continuous positive airway pressure

DGP Deutsche Gesellschaft für Pneumologie und Ventilationsmedizin e.V. (German Respiratory Society)

DIGAB Deutsche interdisziplinäre Gesellschaft für außerklinische Beatmung e.V. (German Interdisciplinary Society for Home Mechanical Ventilation)

ECG Electrocardiogram

EPAP Expiratory positive airway pressure

$\mathrm{FEV}_{1} \quad$ Forced expiratory volume in 1 second

FVC Forced vital capacity

HME Heat and moisture exchange

HMV Home mechanical ventilation

IPPB Intermittent positive pressure breathing

KRINKO Kommission für Krankenhaushygiene und Infektionsprävention (Commission for Hygiene in Hospital and the Prevention of Infection)

LTOT Long-term oxygen treatment

MDI Metered-dose inhaler

MI-E Mechanical Insufflator-Exsufflator

MRP Multidrug-resistant pathogen

NIV Non-invasive ventilation

NMD Neuromuscular diseases

$\mathrm{O}_{2} \quad$ Oxygen

OHS Obesity hypoventilation syndrome

OSAS Obstructive sleep apnoea syndrome

$\mathrm{PaCO}_{2} \quad$ Partial pressure of arterial carbon dioxide

$\mathrm{PaO}_{2} \quad$ Partial pressure of arterial oxygen

PCF Peak cough flow

$\mathrm{PCO}_{2} \quad$ Partial pressure of carbon dioxide

PEEP Positive end-expiratory pressure

$\mathrm{PO}_{2} \quad$ Partial pressure of oxygen

$\mathrm{PtcCO}_{2} \quad$ Partial pressure of transcutaneous carbon dioxide

REM Rapid eye movement

RERA Respiratory effort-related arousal

SGB Sozialgesetzbuch (Social Security Code)

SMI Soft mist inhaler

$\mathrm{SpO}_{2} \quad$ Oxygen saturation

SRI Severe Respiratory Insufficiency Questionnaire

VC Vital capacity 